Chem. Ber. 117, 2963- 2987 (1984)

\title{
Über das cis-Glycol und das Epoxid des Benzvalens
}

\author{
Manfred Christl*, Hartmut Leininger ${ }^{1)}$ und Petra Kemmer \\ Institut fur Organische Chemie der Universităt Würburg, ' \\ Am Hubland, D-8700 Wurzburg
}

Eingegangen am 13. Oktober 1983

\begin{abstract}
Benzvalen (1) laßt sich mit Standardmethoden in das cis-Glycol 2 umwandein, das seinerseits leicht derivatisierbar ist und glatt das Ditosylat 3, das cyclische Carbonat 4 und die cyclischen Essigsidureorthoester 5 und 6 bildet. Unter Belichten nimmt 2 Thiophenol an der Bicyclo[1.1.0]butan-Zentralbindung zum Thioether 8 auf. Die Solvolyse von 3 in wäßrigem Aceton erbringt endo,endo-Tricyclo[2.2.0.02.6 ]hexan-3,5-diol (11). - Das Epoxid 18 von Benzvalen (1) kann entweder aus den Orthoestern 6 uber die Stufe des trans-Chlorhydrinacetats 17 oder, und dies ist fur prăparative Zwecke die Methode der Wahl, direkt aus 1 mit $N$-Benzoylpercarbamidsăure hergestellt werden. Seine Thermolyse fuhrt zunăchst zu 2-Oxabicyclo[3.2.0]hepta-3,6-dien (19), das teilweise in Oxepin (20)/Benzoloxid (21) ubergeht. Beim Zerfall von 3,4-dideuteriertem 18 landen die Marken in den Positionen 5 und 6 von 19. Wenn 18 in hoher Konzentration thermolysiert wird, findet in erheblichem $\mathrm{Maß}$ die Diels-Alder-Addition von 19 an 21 zu einem pentacyclischen Produkt 22 statt. Essigsăure und unter Lichteinwirkung auch Thiophenol bringen aus 181 : 1-Addukte vom Tricyclo[2.2.0.0 $0^{2,6}$ ]hexan-Typ (29 bzw. 28) hervor. Schon beim leichten Erwarmen lagert sich das Diolmonoacetat 29 in das Bicyclo[2.1.1] hex-2-en-Derivat $30 \mathrm{um}$, das bei $130^{\circ} \mathrm{C}$ sejnerseits zum Bicyclo[3.1.0]hex-2-en-Abkömmling 31 isomerisiert. Mit $\mathrm{LiAlH}_{4}$ können aus 18 je nach Losungsmittel und Temperatur entweder fast reines Tricylco[3.1.0.0 2.6] hexan-3-ol (32) oder ein 1: 1-Gemisch aus 32 und endo-Tricyclo[2.2.0.0 2.6] hexan-3-ol (33) erhalten werden. Aufgrund der NMR-Daten der zugehorigen Methylether 34 und 35 erweist sich eine Konstitutionszuordnung in der Literatur als revisionsbedürftig.
\end{abstract}

\section{On the cis-Glycol and the Epoxide of Benzvalene}

By means of standard methods, benzvalene (1) can be transformed into the cis-glycol 2, from which derivatives such as the ditosylate 3 , the cyclic carbonate 4 , and the cyclic orthoacetates 5 and 6 are readily prepared. Irradiation causes thiophenol to add across the central bond of the bicyclo. [1.1.0]butane moiety of 2 with formation of the thioether 8. The solvolysis of 3 in aqueous acetone leads to endo,endo-tricyclo[2.2.0.0 2.6 hexane-3,5-diol (11). The epoxide 18 of benzvalene (1) may be synthesized starting either from the ortho esters 6 via the trans-chlorohydrin acetate 17 or directly from 1 with $N$-benzoylpercarbamidic acid, the latter reaction being the better one. Initially, the thermolysis of 18 produces 2-oxabicyclo[3.2.0]hepta-3,6-diene (19) which in part isomerizes to oxepin (20)/benzene oxide (21). In the thermal decomposition of 3,4-dideuterated 18, the labeis end up in positions 5 and 6 of 19 . If 18 is thermolyzed in high concentration 19, and 21 undergo Diels-Alder addition to a considerable extent to form the pentacyclic product 22. Epoxide 18 adds acetic acid and, under irradiation, thiophenol resulting in $1: 1$ adducts of the tricyclo[2.2.0.0 2.6 Jhexane type ( 29 and 28 , respectively). On slight warming, the diol monoacetate 29 rearranges to the corresponding bicyclo[2.1.1] hex-2-ene derivative 30 , which is converted into its bicyclo[3.1.0]hex-2-ene isomer 31 at $130^{\circ} \mathrm{C}$. Depending on the solvent and the temperature, $\mathrm{LiAlH}_{4}$ reduces 18 either to almost pure tricyclo[3.1.0.0 2.6] $\mathrm{hexan-3-ol}$ (32) or to

(C) Verlag Chemie GmbH, D-6940 Weinheim, 1984

$0009-2940 / 84 / 0909-2963 \$ 02.50 / 0$ 
a 1:1 mixture of 32 and endo-tricyclo[2.2.0.0 $0^{2.6}$ ]hexan-3-ol (33). On the basis of the NMR data of the corresponding methyl ethers 34 and $\mathbf{3 5}$, a structure assignment in the literature has to be revised.

Den Reaktionen olefinischer Doppelbindungen mit Oxidationsmitteln kommt große synthetische Bedeutung zu. Um die präparativen Moglichkeiten zu erkunden, haben wir die klassischen Oxidationen am Benzvalen (1) untersucht. Insbesondere stand die Frage im Mittelpunkt, welche Reaktionsbedingungen das Bicyclo[1.1.0]butan-System uberstehen warde. Als Olefin mit einem niedrigen lonisationspotential ${ }^{2)}$ tritt 1 bereitwillig mit Elektrophilen aller Art in Wechselwirkung ${ }^{3)}$ und sollte daher auch gut oxidierbar sein. Kürzlich haben wir uber die Addition von Ozon ${ }^{4)}$ und

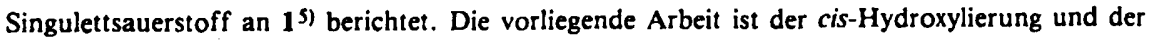
Epoxidation gewidmet $\theta$.

\section{A. Synthese und Reaktionen des cis-Glycols 2}

Bereits mit Kaliumpermanganat unter Standardbedingungen erhielten wir aus 1 das cis-Glycol 2, wobei die maximale Ausbeute von $42 \%$ bei $-15^{\circ} \mathrm{C}$ im 10 -mmol-Maßstab erzielt wurde. Erhöhung der Ansatzgroße führte zur Ausbeuteminderung. Dagegen konnte die Umsetzung von 1 nach der Methode von Sharpless et al. ${ }^{7}$ mit tert-Butylhydroperoxid und katalytischen Mengen Osmiumtetroxid bei einer Ausbeute von $54 \%$ an 2 auch auf großere Ansătze übertragen werden. Ein weiterer Weg zu 2 besteht in der Behandlung des 1,2-Dioxetan-Derivats, das sich aus 1 und Singulettsauerstoff bildet, mit Triphenylarsan in Gegenwart von Feuchtigkeit ${ }^{\text {s). }}$<smiles>C1=CC2C3C1C1C2C31</smiles>

1

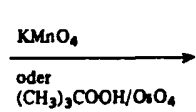

$\left(\mathrm{CH}_{3}\right)_{3} \mathrm{COOH} / \mathrm{OrO}_{4}$<smiles>OC1C2C3C4C1C1C2C3C41O</smiles>

2

Mit Tosylchlorid in Pyridin konnten wir 2 mit 69\% Ausbeute in das Ditosylat 3 uberführen. Dagegen war das Monotosylat von 2 auch bei Verwendung von unterschüssigem Reagenz nicht nachweisbar. Aus Phosgen und 2 bildete sich in Pyridin glatt (72\% Ausbeute) der cyclische Kohlensäureester 4 . Die Darstellung der Orthoester 5 und 6, jeweils ein Stereoisomerengemisch, gelang mit 1,1,1-Triethoxy- bzw. 1,1,1-Trimethoxyethan in Gegenwart einer katalytischen Menge Benzoesăure. Konstitution und Stereochemie aller Produkte werden durch die NMR-Spektren belegt (Abschnitt F).
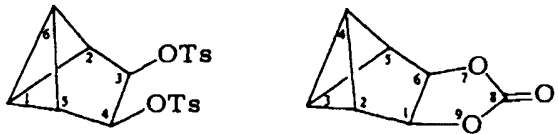

$3\left\langle\mathrm{Ts}=\mathrm{O}_{2} \mathrm{~S}-\mathrm{C}_{6} \mathrm{H}_{4}-4-\mathrm{CH}_{3}\right)$

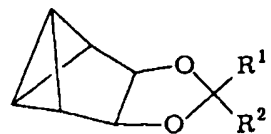

4

\begin{tabular}{l|ll} 
& $\mathrm{R}^{2}$ & $\mathbf{R}^{2}$ \\
\hline $\mathbf{5 a}$ & $\mathrm{OC}_{2} \mathrm{H}_{5}$ & $\mathrm{CH}_{3}$ \\
$\mathbf{5 b}$ & $\mathrm{CH}_{3}$ & $\mathrm{OC}_{2} \mathrm{H}_{5}$ \\
$\mathbf{6 a}$ & $\mathrm{OCH}_{3}$ & $\mathrm{CH}_{3}$ \\
$\mathbf{6 b}$ & $\mathrm{CH}_{3}$ & $\mathrm{OCH}_{3}$
\end{tabular}

Besonders die Bildung von 5 und 6 deutet auf eine uberraschende Resistenz ihres Bicyclo[1.1.0]butan-Systems gegenuber Säuren hin. Im Gegensatz zu 2 kann Bicyclo[1.1.0]butan-endo,endo2,4-dimethanol (7) nur unter Schwierigkeiten acyliert werden 4), wohl weil 7 und seine Derivate die Aciditat der Reaktionsgemische bei Anwendung der Pyridinmethode nicht vertragen. Die variierende Saureempfindlichkeit verschiedener Bicyclo[1.1.0]butan-Abkommlinge hat ihre Ursache im 
Interplanarwinkel der beiden Dreiringe. Anhand der Photoelektronenspektren war gezeigt worden, daß parallel zur Vergroßerung dieses Winkels die Energie des in der Zentralbindung lokalisierten HOMOs anwăchst ${ }^{8.9}$, womit eine Erhobung der Reaktivităt des $\sigma$-Systems gegenubuer Elektrophilen einhergehen sollte. Für die als elektrophil geltenden Thiylradikale wurde dieser $\mathrm{Zu}$ sammenhang experimentell bestătigt ${ }^{9}$.<smiles>OCC1C2C3C(CO)C2C13</smiles>

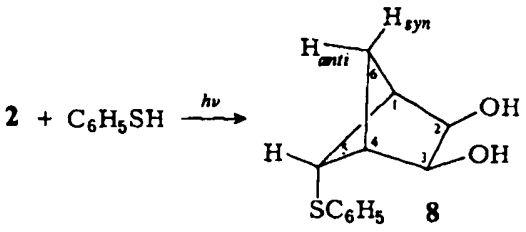

Weil in 2 und 7 besagter Interplanarwinkel unterschiedlich groß ist - in $\mathbf{2}$ kleiner als normal wegen der Zweikohlenstoffbrucke und in 7 großßer wegen der wechselseitigen Abstoßung der Hydroxymethylgruppen - , nehmen diese Substanzen Thiophenol unterschiedlich rasch auf, wobei aus Analogiegrunden der Mechanismus uber das Phenylthiylradikal angenommen werden darf ${ }^{10,11}$. Bei 7 lauft die Reaktion bei Raumtemperatur exotherm in zwei Minuten vollständig $\mathrm{ab}^{4}$. Dagegen ist 2 gegenuber Thiophenol bei $20^{\circ} \mathrm{C}$ inert und erst die Belichtung erbringt rasch das $1: 1$-Addukt 8 mit $67 \%$ Ausbeute. In Übereinstimmung mit Befunden von Szeimies et al. ${ }^{10}$ addiert sich das Radikal von der răumlich zugănglicheren Seite, also trans-orientiert zu den Hydroxylgruppen an die Bicyclo[1.1.0]butan-Zentralbindung unter Inversion an C-1.

Diese Stereochemie beweist das ${ }^{1} \mathrm{H}$-NMR-Spektrum von 8, in dem 5-H ein scharfes Triplett bei $\delta=3.46 \mathrm{mit} J_{1,5}=2.7 \mathrm{~Hz}$ verursacht. Hătte C-5 die andere Konfiguration, mußße eine große

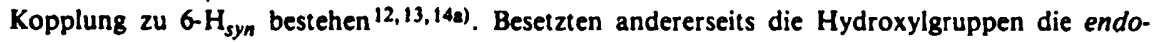
Positionen, sollte eine meßbare Fernkopplung $J_{2,5}$ wegen der W-formigen Bindungsanordnung ${ }^{14 a)}$ zwischen diesen Kernen beobachtbar sein. Tatsăchlich läßt sich eine solche Wechselwirkung zwischen 2,3-H und 6- $\mathrm{H}_{\text {anti }}$ für die in 8 angegebene Konfiguration erwarten und aus der Breite des Signals des letzteren auch ablesen.

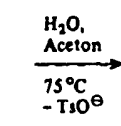<smiles>CCOC1C2C3CC4C3C4C12</smiles><smiles>COC1C2CC3C2C([O+])C31</smiles><smiles>CCOc1c2cccc1C2</smiles>
10 $\mid \begin{aligned} & +\mathrm{H}_{2} \mathrm{O} \\ & -\mathrm{H}^{\oplus}\end{aligned}$<smiles>OC1C2C3CC2C2C1C32</smiles>

11<smiles>CC(O)C1C2CCC1C2</smiles>
$\mathrm{OH}$<smiles>C#CC1C2C3C(O)C2C13</smiles>

12<smiles>OC1C2CC3CC2C(O)C31</smiles>

13

Das Ditosylat 3 haben wir in 60proz. waßrigem Aceton in Gegenwart eines tertiăren Amins zur Abpufferung der entstehenden Saure solvolysiert und mit 38\% Ausbeute

Chem. Ber. 117(1984) 
das endo, endo-Tricyclo[2.2.0.0 2,6] Thexan-3,5-diol (11) isoliert. Sein ${ }^{1}$ H-NMR-Spektrum, das Konstitution und Stereochemie beweist, ist in Abschnitt F diskutiert. Für die Abdissoziation der ersten Tosylatgruppe sind zwei Möglichkeiten denkbar: erstens der $S_{N} 1-P r o z e ß z u g$ und zweitens der $S_{N} 1$-Prozeß unter Nachbargruppenbeteiligung, d. h. mit $[1,2]$-Wanderung der antiperiplanar angeordneten Bicyclo[1.1.0]butan-Brückenkopf-CH-Gruppe, was sofort zu 10 mit einheitlicher endo-Stereochemie führen müßte. Von 9 ausgehend sollte die Wagner-Meerwein-Umlagerung dagegen das Kation 10 als Stereoisomerengemisch liefern, da beide Bicyclo[1.1.0]butan-Brückenkopf-CH-Gruppen mit ähnlicher Triebkraft wandern können. Der nucleophile Angriff eines Wassermoleküls muß aufgrund der Konstitution des Endprodukts den Monoalkohol 13 hervorbringen, der als Tosylat eines Cyclopropylcarbinol-Derivats leicht dissoziieren sollte. So gebildetes 12, das im Formelschema wie das verwandte Kation 10 in klassischer und nichtklassischer Schreibweise ${ }^{(5)}$ dargestellt ist, nimmt Wasser zum Endprodukt 11 auf.

Wasser als Nucleophil scheint selektiv die Zweikohlenstoffbrucke von 6-Bicyclo[2.1.1]hex-2enyl-Kation-Derivaten von der Ruckseite her anzugreifen. Dagegen bevorzugen Halogenidionen das kationische Zentrum der Einkohlenstofforucke, woraus Bicyclo[2.1.1]hex-2-en-Derivate hervorgehen 16,17). Aufgrund der Thermolabilität des Monoacetats 29 (Abschnitt D) von 11 liegt die Vermutung nahe, daß unter kinetischer Steuerung Tricyclo[2.2.0.0.6.6 hexan-Derivate entstehen. Ist deren Bildung aber reversibel, was wir bei guten Abgangsgruppen $\left(\mathrm{Br}{ }^{\ominus}, \mathrm{Cl}^{\ominus}, \mathrm{CH}_{3} \mathrm{CO}_{2}{ }^{\ominus}\right.$ ) schon bei niedriger Temperatur für möglich halten, resultieren Bicyclo[2.1.1]hex-2-en-Derivate (thermodynamischer Reaktionsabschluß).

\section{B. Synthesen des Epoxids 18}

Zunächst scheiterten alle Versuche, die Doppelbindung von 1 auf konventionelle Weise mit Peressigsăure oder $m$-Chlorperbenzoesäure zu epoxidieren, auch als Natriumcarbonat oder Natriumdihydrogenphosphat/Dinatriumhydrogenphosphat zugesetzt wurden, um die frei werdenden Carbonsäuren abzupuffern. Wir wandten deshalb eine Syntheseroute an, die Halogenhydrinester als Vorstufen benutzt ${ }^{18)}$ und mit der empfindliche Oxirane wie Arenoxide ${ }^{19)}$ und Epoxynucleoside ${ }^{20)}$ bereitet wurden. Die Ausgangsmaterialien erhält man unter anderem aus cis-Glycolen über Orthoester vom Typ 5, 6, wobei Chlortrimethylsilan ${ }^{192,21)}$ und Chlortriphenylmethan ${ }^{22)}$ als Reagenzien zum Einsatz kommen. Die Behandlung von 6 mit Chlortrimethylsilan fuhrte nicht zum Erfolg, jedoch erbrachte die Einwirkung von Chlortriphenylmethan mit $56 \%$ Ausbeute das trans-Chlorhydrinacetat 17, verunreinigt mit einem kleinen Anteil seines umgelagerten Isomeren 16.

Chlortriphenylmethan generiert aus 6 wohl zunächst das Dialkoxycarbeniumsalz 14, das durch eine $\mathrm{S}_{\mathrm{N}}$ 2-Reaktion des Chloridions in 17 ubergeht (Weg a). Daneben tritt in geringem Ausmaß die Wagner-Meerwein-Umlagerung zu 15 ein (Weg b), wobei die einheitliche Stereochemie des Endprodukts 16 nahelegt, daß die [1,2]-Wanderung des zum Heterocyclus trans-ständigen C-3 gleichzeitig mit der Öfnung der CO-Bindung ablauft. Der Rückseitenangriff des Chloridions am kationischen Zentrum der Einkohlenstoffbrücke in 15 ergibt zwanglos 16 (vgl. Diskussion zur Bildung von 11). Wie beim Episulfoniumion, das bei der Addition von $N$-(Chlorthio)succinimid an 1 durchlaufen wird $^{17}$, hălt sich auch im Falle von 14 die Umlagerung in die Bicyclo[2.1.1]hex-2-en- 
Reihe im Vergleich zur beabsichtigten $S_{N} 2$-Reaktion zum Tricyclo[3.1.0.0 2,6]hexanDerivat in Grenzen.

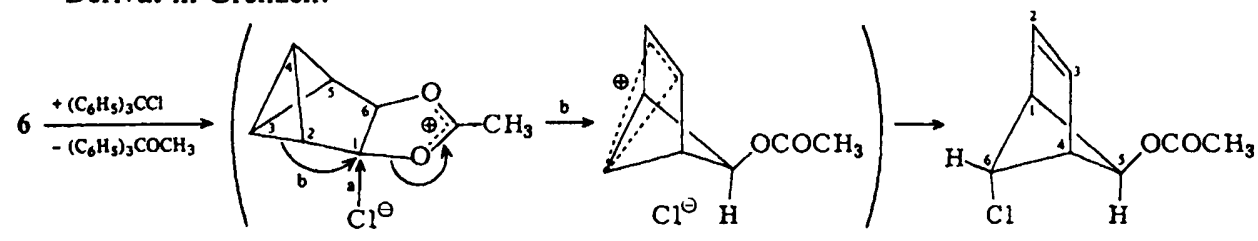

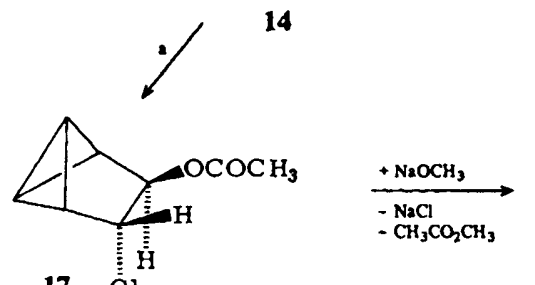

15<smiles>C1C2C3C4C1C1C2C3C41</smiles>

18

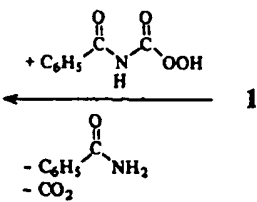

Aus der Umsetzung von 17 mit Natriummethoxid in Triglyme erhielten wir Benzvalenoxid (18) mit ca. 50\% Ausbeute. Durch Umesterung entsteht dabei zuerst aus 17 das Alkoholat des trans-Chlorhydrins, in dem dann die intramolekulare $\mathrm{S}_{\mathrm{N}} 2$-Reaktion $\mathrm{zu}$ 18 abläuft. Die so bewiesene Existenzfähigkeit von 18 veranlaßte uns, nach einem Reagenz zu suchen, das 1 direkt in $\mathbf{1 8}$ umwandelt, ohne Folgereaktionen des letzteren auszulösen. $N$-Benzoylpercarbamidsäure erschien wie maßgeschneidert. Höft und Ganschow ${ }^{23)}$ hatten sie erstmals hergestellt, zur Synthese von Epoxiden und Oxaziridinen aus Olefinen bzw. Schiffschen Basen eingesetzt und zur Präparation von săureempfindlichen Verbindungen dieses Typs empfohlen, da als Coprodukte nur Benzamid und Kohlendioxid anfallen. Spăter wurde die Epoxidierungspotenz dieser Persăure wiederentdeckt ${ }^{24)}$ und auch zur Herstellung der labilen 2-Azidooxirane genutzt ${ }^{25)}$. Tatsăchlich erhielten wir aus 1 und $N$-Benzoylpercarbamidsăure eine 54proz. Ausbeute an 18. Bei der Reaktion erwies sich Wasserausschluß als notwendig, da sich 18 schon mit Wasser rasch in nicht näher untersuchter Weise verändert. Mit den üblichen Percarbonsăuren können inerte Bedingungen, wie sie für 18 und andere empfindliche Epoxide $^{25)}$ essentiell sind, auch durch Zusătze nicht geschaffen werden.

Konstitution und Stereochemie von $16-18$ werden durch die NMR-Daten gestützt. Jene von 17 und 18 sind im Abschnitt F diskutiert. Im ' H-NMR-Spektrum von 16 beweist die im Gegensatz zu $J_{1,5}=2.2 \mathrm{~Hz}$ nicht aufgeloste Kopplung $J_{1,6}$ die unterschiedliche Stereochemie an C-6 und C-5 ${ }^{12)}$. Daß die Acetoxygruppe endo- und der Chlorsubstituent anti-ständig angeordnet sind, folgt aus dem Vergleich mit den chemischen Verschiebungen von endo, anti-5,6-Dichlorbicyclo[2.1.1] hex-2-en 16b,26). Wahrend die drei restlichen Signale recht ahnliche Werte aufweisen, erscheint das Triplett, das jeweils 5-H zuzuordnen ist, im Spektrum von 16 um 0.32 ppm bei tieferem Feld, wie es beim Ersatz eines Chlor-durch einen Acetoxysubstituenten erwartet werden muß 27a).

\section{Die Thermolyse des Epoxids 18}

Eine 15proz. $\left[D_{6}\right]$ Benzollosung von $18 \mathrm{mit}$ Zusătzen an Cyclohexan und Tetramethylethylendiamin und den Verunreinigungen Chloroform und Tetrahydrofuran wurde 
im abgeschmolzenen NMR-Rohr bei $145^{\circ} \mathrm{C}$ thermolysiert. Nach $11 \mathrm{~h}$ war 18 vollstăndig verschwunden und ein 7:3-Gemisch aus 2-Oxabicyclo[3.2.0]hepta-3,6-dien (19) und Oxepin/Benzoloxid (20/21) mit wenigen Prozent Ausbeute entstanden. Das restliche Material verfügt im NMR-Spektrum uber keine Olefinbanden und erwies sich'als zumindest aus zwei 18-Einheiten hervorgegangen. Die ${ }^{13} \mathrm{C}$-chemischen Verschiebungen eines Vakuum-destillierbaren Anteils deuten auf das Strukturelement des Dialkohols 11. Angesichts der Reaktionsprodukte 28 und 29 von Thiophenol und Essigsaure mit 18 könnten diese Verbindungen durch eine von Spuren einer Protonen-aktiven Substanz $\left(\mathrm{H}_{2} \mathrm{O}\right.$ ?) ausgeloste Oligomerisierung gebildet worden sein. Ein OH-Signal im ' $\mathrm{H}-\mathrm{NMR}$ Spektrum stützt diese Vermutung.

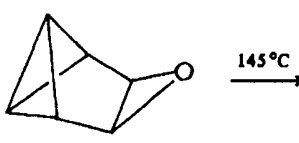

18
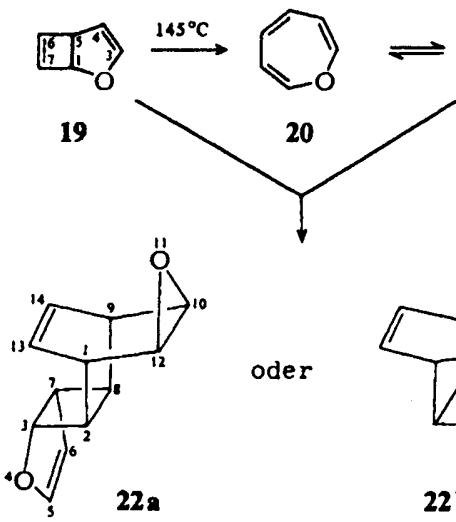

oder

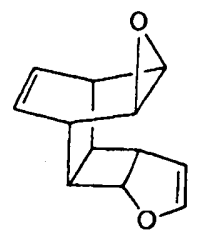

$22 b$

Die Verbindungen 20/2128,29) sowie ihr Photoprodukt 1929-31) wurden anhand ihrer bekannten ${ }^{1} \mathrm{H}$-NMR-Spektren identifiziert. Wir halten die fur 19 publizierte Zuordnung ${ }^{32}$ ) fur korrekt, jedoch brachten Entkopplungsexperimente zwei weitere Fernkopplungen an den Tag. Besonders erwahnt sei $J_{4,7}=0.4 \mathrm{~Hz}$, fur deren Große die Zickzack-Anordnung ${ }^{14 a)}$ der Bindungen uber $\mathrm{C}-1$ verantwortlich sein durfte. Tab. 1 enthalt alle Daten. Von Lit. ${ }^{32)}$ abweichende chemische Verschiebungen beruhen auf dem unterschiedlichen Losungsmittel. Im ${ }^{13} \mathrm{C}$-NMR-Spektrum charakterisiert der Wert $\delta=105.0$ die $\beta$-Position (C-4) einer Enolethereinheit.

Tab. 1. 'H-NMR-Chemische Verschiebungen ( $\delta$-Werte, Diagonalelemente) und Kopplungskonstanten (Abs Jlutwerte in Hz, Nichtdiagonalelemente) von 2-Oxabicyclo[3.2.0]hepta-3,6-dien (19) in $C_{6} D_{6}$

\begin{tabular}{llllllll}
\hline & & $1-\mathrm{H}$ & $3-\mathrm{H}$ & $4-\mathrm{H}$ & $5-\mathrm{H}$ & $6-\mathrm{H}$ & $7-\mathrm{H}$ \\
\hline & $1-\mathrm{H}$ & 5.13 & 0.8 & $2)$ & 3.8 & 2.7 & a) \\
& $3-\mathrm{H}$ & & 6.24 & 3.0 & 0.8 & a) & a) \\
19 & $4-\mathrm{H}$ & & & 4.96 & 2.8 & a) & 0.4 \\
& $5-\mathrm{H}$ & & & & 3.44 & a) & 1.8 \\
& $6-\mathrm{H}$ & & & & & 6.34 & 2.7 \\
& $7-\mathrm{H}$ & & & & & & 5.79 \\
\hline
\end{tabular}

a) $<0.4 \mathrm{~Hz}$. 
Bei höherer Konzentration (ca. $70 \% 18$ in $\mathrm{C}_{6} \mathrm{D}_{6}$ ) verlief die Thermolyse sauberer. $\mathrm{Zu}$ 43\% bildete sich 19 und mit 22\% Ausbeute fiel eine Festsubstanz an, die aufgrund von je zwolf Signalen in ${ }^{1} \mathrm{H}$ - und ${ }^{13} \mathrm{C}$-NMR-Spektren unsymmetrisch aus zwei $\mathrm{C}_{6} \mathrm{H}_{6} \mathrm{O}$ Einheiten aufgebaut sein muß. Alle Daten sprechen dafur, daß ein Stereoisomeres der Konstitution 22 vorliegt. Die trans-Anellierung am Cyclobutanring folgt aus dem groBen Unterschied der Kopplungskonstanten $J_{2,8}$ und $J_{3,7}$ einerseits und $J_{2,3}$ und $J_{7,8}$ andererseits, wobei sich die Zuordnung auf Entkopplungsexperimente im $360-\mathrm{MHz}^{-1} \mathrm{H}$ NMR-Spektrum stutzt. Bezuglich der Angliederung des Epoxidrings bieten die Spektren keine Anhaltspunkte, jedoch erscheint aufgrund der Bildungsreaktion die in 22a und $22 \mathrm{~b}$ angegebene Stereochemie am wahrscheinlichsten. Offensichtlich handelt es sich um ein Diels-Alder-Addukt der Cyclobutendoppelbindung von 19 an das Diensystem von 21. Da die höhere Konzentration der Komponenten den bimolekularen Prozeß fordert, ist das Nichtauffinden dieser Verbindung bei der Thermolyse der verdunnteren 18-Losung verständlich. Die H-Atome an $C-13$ und $C-14$ in 22a bedrăngen den Cyclobutanring weniger als $10-\mathrm{H}$ und $12-\mathrm{H}$ in $22 \mathrm{~b}$, was bereits fur die Ubergangszustände der Bildung dieser Diastereoisomeren gelten dürfte. Wir geben daher 22a den Vorzug vor 22b.

Es ist bekannt, daß sich im Rahmen von [4+2]-Cycloadditionen 19 mit der Cyclobutendoppelbindung an 2,5-Dimethyl-3,4-diphenylcyclopentadienon und $\alpha$-Pyron ${ }^{31)}$, ferner 21 mit dem Diensystem an Maleinsăureanhydrid, Acetylendicarbonsaure-dimethylester ${ }^{28,29)}$ sowie Azodicarbonsăure-bis(2,2,2-trichlorethylester) ${ }^{33)}$ anlagern. Daher uberrascht die Bildung von 22 nicht, wenn auch die elektronischen Verhältnisse für die Additionen 19 an 21 nicht optimal erscheinen.

Die Untersuchung des Thermolyseverlaufs mit Hilfe der ${ }^{1} \mathrm{H}$-NMR-Spektroskopie laßt vermuten, daß 20/21 aus 19 entsteht. Jedoch ist aufgrund des ungunstigen Signal-

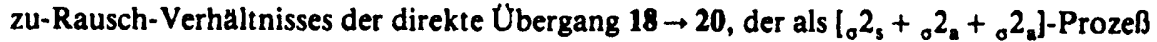
erlaubt wäre ${ }^{34)}$, nicht ausgeschlossen. Wie ein unabhängiger Versuch bei $150^{\circ} \mathrm{C}$ in entgastem $\mathrm{C}_{6} \mathrm{D}_{6}$ zeigte, lagert sich 19 in 20/21 um, ohne daß dabei die Weiterisomerisierung zu Phenol eintritt. Fruher ${ }^{29)}$ hatte man nur Phenol aus der Thermolyse von 19 bei $150-160^{\circ} \mathrm{C}$ erhalten. Vermutlich waren damals Protonen-aktive Substanzen nicht rigoros genug ferngehalten worden. Das 1,3-Dimethylderivat von 19 ist durch Belichten von 2,7-Dimethyloxepin erhalttlich und soll schon unter den Bedingungen der Aufarbeitung die teilweise Ruckisomerisierung erleiden ${ }^{35)}$.

Im Vergleich zur Thermolyse in Losung verschob sich das Produktverhalltnis drastisch, als $18 \mathrm{im}$ Vakuum durch ein auf $250^{\circ} \mathrm{C}$ erhitztes Quarzrohr geleitet wurde. Weit überwiegendes Hauptprodukt war jetzt 20/21, und nur ca. 3\% betrug der Anteil von 19. Es scheint ein verbreitetes Phänomen zu sein, daß bei Gasphasenthermolysen Zwischenstufen, die in Losung oft in hoher Konzentration auftreten, nicht oder kaum nachweisbar sind. Wahrscheinlich kann in der Gasphase die Uberschußenergie - jene von 19 durfte mindestens $60 \mathrm{kcal} / \mathrm{mol}$ betragen, wenn man abgeschătzte Aktivierungsund Reaktionsenthalpien addiert - nicht rasch genug abgegeben werden. Ausgehend von einem hoch Schwingungs-angeregten Zustand uberwindet das System dann die năchste Aktivierungsbarriere leicht.

Beim Erhitzen des Dideuteroderivats 18a bildete sich hauptsachlich 19a, das die Marken an C-5 und C-6 trăgt. Damit verlauft die Thermolyse von 18 wie die vom 
Stammkohlenwasserstoff Tetracyclo[4.1.0.0 $2,4 \cdot 0^{3,5}$ ]heptan ${ }^{36)}$. Eine Entscheidung zugunsten einer mechanistischen Alternative - die wichtigsten sind der Einstufenprozeß vom $\left[{ }_{\sigma} 2_{s}+{ }_{\sigma} 2_{a}+{ }_{\sigma} 2_{a}\right]-T_{y p}{ }^{34)}$ und die Mehrstufenreaktion uber die Diradikale 23 und 24 sowie das trans, cis, cis-Oxepin 25 - kann bisher nicht getroffen werden. Die thermische Stabilitat von 18 ist der seines Schwefelanalogen 7-Thiatetracyclo[4.1.0.0 2,4.03,5]heptan ${ }^{17}$ vergleichbar. Allerdings sind bei dessen Zerfall keine $\mathrm{C}_{6} \mathrm{H}_{6}$ S-Isomeren beobachtbar sondern nur Benzol. 1-Phenylazepin entsteht bei $150^{\circ} \mathrm{C}$ aus 7-Phenyl-7-azatetracyclo[4.1 $0.0^{2,4} \cdot 0^{3,5}$ heptan ${ }^{1,37)}$.

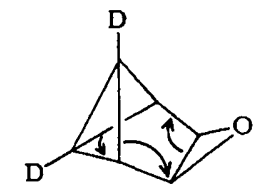

$18 \mathbf{a}$

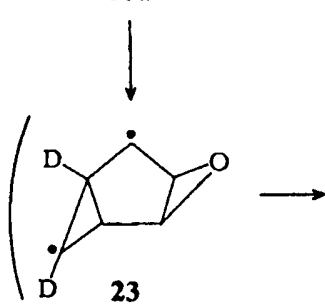

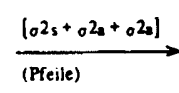

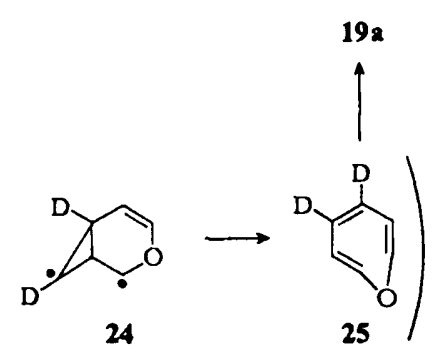

\section{Die Addition von Thiophenol und Essigsäure an 18}

Tetracyclo[4.1 $0.0^{2,4} \cdot 0^{3,5}$ ]heptan nimmt Thiophenol je nach den Bedingungen in einer polaren Addition an einer peripheren oder in einer Radikalreaktion an der zentralen Bicyclo[1.1.0]butan-Bindung auf ${ }^{11}$ ). Bei 7-Thiatetracyclo[4.1.0.0 $0^{2.4} .0^{3.5}$ ]heptan dagegen lăuft ein Radikalprozeß ab, der am Schwefelatom einsetzt ${ }^{17}$. Einen dritten Reaktionstyp beobachteten wir jetzt bei 18. Zwar erwies sich 18 bei Raumtemperatur gegenuber Thiophenol als inert, aber durch Belichten konnte eine Umsetzung ausgelöst werden, die mit 76\% Ausbeute 27 und 28 im Verhaltnis 5:95 erbrachte. Obwohl weder die Trennung noch die Reinigung des Gemisches gelang, belegt das charakteristische ' $\mathrm{H}$ NMR-Spektrum Konstitution und Stereochemie von 28 (Abschnitt F). Dagegen sprechen für 27 nur zwei verbreiterte Singuletts bei $\delta=3.32$ und 3.78, die allerdings fur ein trans-3,4-disubstituiertes Tricyclo[3.1.0.0 2,6]hexan-Derivat typisch sind, wie das Beispiel 17 unterstreicht.

Offenbar initiiert die Belichtung hier keinen Radikalprozeß, sondern die Anlagerung eines Protons an das Sauerstoffatom von 18. Vermutlich ist Thiophenol im angeregten Zustand eine stărkere Săure, als im Grundzustand. Fur Phenol und einige andere Molekule hat man diesen Sachverhalt bewiesen ${ }^{38}$. Wie schon 14 sieht sich das Kation 26 einer Konkurrenzsituation gegenuber. Zum einen kann Thiophenolat den Dreiring im Sinne einer $S_{\mathrm{N}} 2$-Reaktion zu 27 offnen, was der gewohnlichen saurekatalysierten $\mathrm{HX}$ Anlagerung an Epoxide entspricht. Zum zweiten fuhrt die leichter eintretende Wagner- 
Meerwein-Umlagerung zum nichtklassischen Carbeniumion 12, das Thiophenolat zu 28 aufnimmt. Wegen der einheitlichen Stereochemie von 28 wandert (Weg b) nur das zum Sauerstoffatom trans-stăndige Bicyclo[1.1.0]butan-Bruckenkopf-Kohlenstoffatom, was wie bei der Bildung von 16 darauf hinweist, daß ein freies Tricyclo[3.1.0.0 2.6]hex-3yl-Kation (vgl. 9) nicht auftritt, da dort zwei Wanderungsmoglichkeiten mit unterschiedlichen stereochemischen Konsequenzen bestehen.

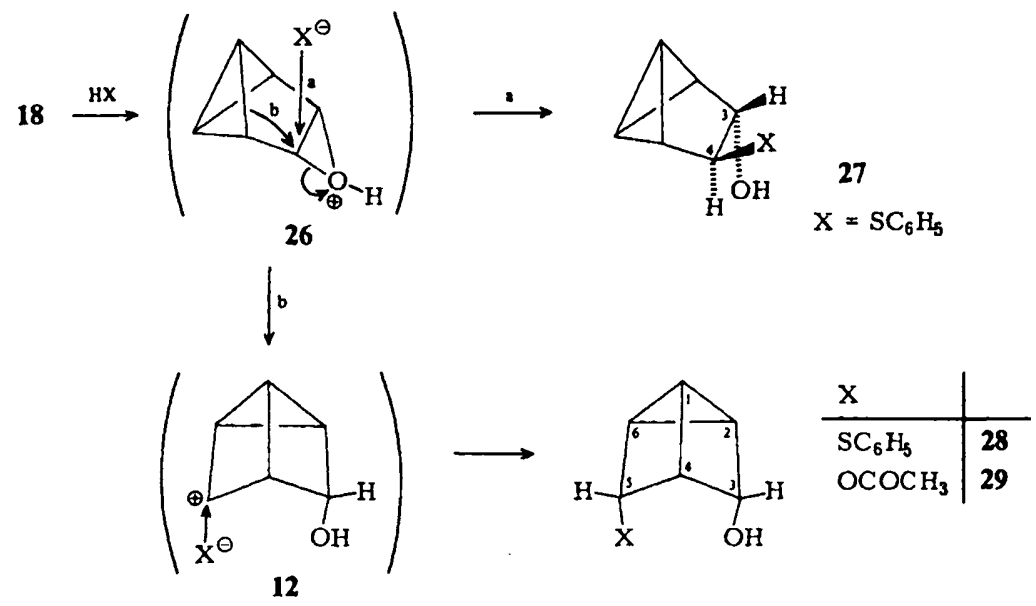

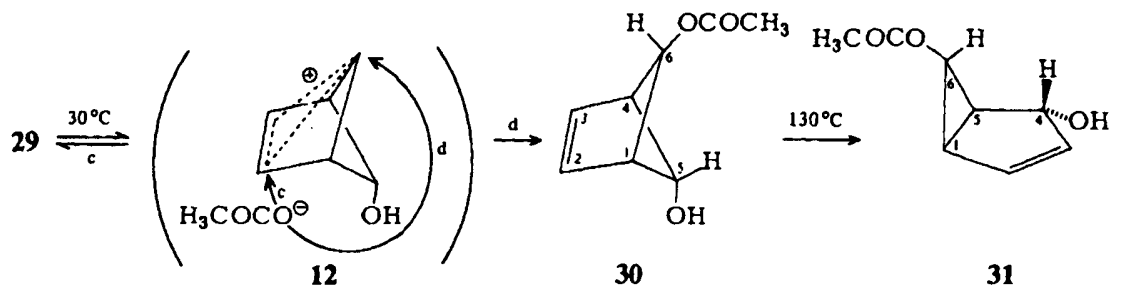

Aus Essigsăure und 18 ging mit 73\% Ausbeute das umgelagerte Addukt 29 hervor. Im Vergleich zum Thiophenolfall bewirkt die hőhere Acidităt der Essigsăure den glatten thermischen Reaktionsablauf bei Raumtemperatur und die geringere Nucleophilie des Acetations das ausschließliche Beschreiten des Umlagerungswegs $b$.

Das Acetat 29 erwies sich als thermolabil und ging schon bei $30^{\circ} \mathrm{C}$ mit beachtlicher Geschwindigkeit in das Bicyclo[2.1.1]hex-2-en-Derivat 30 uber, das seinerseits bei $130^{\circ} \mathrm{C}$ zum Bicyclo[3.1.0]hex-2-en-Abkommling 31 isomerisierte. Ausgehend von 18 isolierten wir 31 mit $61 \%$ Gesamtausbeute.

Die ${ }^{1} \mathrm{H}$-NMR-Spektren beweisen die endo, endo-Stereochemie von 28 und 29 (Abschnitt F) sowie die endo, anti-bzw. exo, exo-Anordnung der Substituenten in 30 und 31. Wie bei 16 lassen sich die Positionen der Substituenten in 30 durch Vergleich mit den chemischen Verschiebungen von endo, anti-5,6-Dichlorbicyclo[2.1.1] hex-2-en 16b. 26) ableiten. Das Triplett von 5-H $(\delta=5.28)$ und das Singulett von $6-H(\delta=4.61)$ liegen zwischen den entsprechenden Signalen der Referenzverbindung, da relativ zur Wirkung eines Chlorsubstituenten das Proton an einem sekundăren Zen-

Chem. Ber. $/ 17(1984)$ 
trum durch eine OH-Gruppe abgeschirmt und durch eine Acetoxygruppe entschirmt wird 27a). Daraber hinaus zeigt das 5-H-Triplett eine Verbreiterung, welche auf die Kopplung zum austauschenden $\mathrm{OH}-\mathrm{Proton}$ hinweist. Die Stellungen von $\mathrm{OH}$ - und Acetoxygruppe in 31 verraten sich durch die chemischen Verschiebungen der zugehörigen H-Atome. So kommt der Allylposition (4 H) $\delta=4.34$ und der Cyclopropylposition $(6-\mathrm{H}) \delta=3.00 \mathrm{zu}$. Daß die OH-Funktion die 4-Stellung besetzt, folgt aus der Kopplung $(6.4 \mathrm{~Hz})$ des $\mathrm{O}-\mathrm{H}$ mit $4-\mathrm{H}$, die in trockenem $\left[\mathrm{D}_{6}\right.$ ]Dimethylsulfoxid beobachtbar ist. Schließlich erkennt man die exo-Anordnung beider Gruppen an den kleinen Werten ( $<2 \mathrm{~Hz}$ ) der Kopplungen $J_{1,6}, J_{5,6}$ und $J_{4,5}$, womit aufgrund der Karplus-ConroyRegel ${ }^{14 b)}$ die trans-vicinale Beziehung dieser Protonen feststeht.

Verbindungen des Typs 28 und 29 wurden aus den Umsetzungen von Benzvalen (1) mit 4-Phenyl-1,2,4-triazolin-3,5-dion ${ }^{39}$, Chlorsulfonylisocyanat ${ }^{40,41)}$ und Tetracyanethylen ${ }^{41}$ ) sowie bei der Behandlung von endo, anti-5,6-Dichlorbicyclo[2.1.1] hex-2-en, dem Chloraddukt von 1, mit Kaliumcyanid, Kaliumacetat oder auch Silberacetat ${ }^{16 b, 26)}$ erhalten. Das zu 11 und 29 gehorende Diacetat ließ sich unter $\mathrm{HCl}$-Einwirkung in das von $\mathbf{3 0}$ abgeleitete Isomere umlagern, und beide Derivate erbrachten beim Erhitzen das Isomere mit dem Gerust von $31^{166)}$.

Der einfachste Weg von 29 nach 30 führt wohl über die Abdissoziation des Acetations zum Kation 12 und Rekombination der Ionen unter thermodynamischer Lenkung zum stabileren Isomeren 30 (vgl. die Diskussion zu der Bildung von 11). Bei der Umlagerung $30 \rightarrow 31$ dürfte eine konzertierte [1,3]-C-Verschiebung der acetoxysubstituierten Brücke ablaufen. Die von der Theorie geforderte Inversion am wandernden Zentrum ${ }^{34)}$ ist in 31 eingetreten. Daß nur C-6 bei der Umordnung mitwirkt und C-5 unbeteiligt bleibt, dürfte auf den sterischen Einfluß der Substituenten zurückgehen. Sie würden einander im endo,endo-konfigurierten Produkt bedrängen, was vermutlich schon den Ubergangszustand energetisch anhebt.

Die Umlagerung des unsubstituierten Bicyclo[2.1.1]hex-2-ens in Bicyclo[3.1.0]hex-2-en ist bekannt ${ }^{42 a)}$ und wurde auch kinetisch untersucht ${ }^{42 b}$. Gleiches gilt fur einige 5 -substituierte ${ }^{i 5 b, 42 c, 42 d)}$ und 5,6-disubstituierte ${ }^{16 \mathrm{~b})}$ Derivate, welche auch die Stereochemie der [1,3]-C-Wanderung of fenbaren.

\section{E. Die Reduktion von 18 mit komplexen Hydriden}

Die reduktive Öfnung des Epoxidrings von 18 versprach einen Weg zum Tricyclo[3.1.0.0 $0^{2.6}$ hexan-3-ol (32), dem formalen Addukt von Wasser an die Doppelbindung des Benzvalens (1). Von Interesse ist diese Umsetzung deshalb, weil das bisher unbekannte 32 nicht direkt aus 1 bereitet werden kann. Sowohl bei der Oxymercurierung ${ }^{43)}$ als auch bei der Hydroborierung ${ }^{44)}$ attackieren die Reagenzien zuerst das Bicyclo[1.1.0]butan-System von 1. Als Reduktionsmittel für bicyclische Epoxide, die häufig langsam, unvollständig und oft unter Umlagerung reagieren, haben sich Lithiumaluminiumhydrid, Lithiumtriethylborhydrid und Lithium in Ethylendiamin besonders bewährt ${ }^{45}$.

Wahrend das letztere Reduktionsmittel 18 nicht in erhoffter Weise umwandelte, kamen wir mit den ersteren zum Wunschmolekul 32, das allerdings im Gemisch mit dem umgelagerten Isomeren 33 anfiel. Ein ăhnliches Ergebnis hatte die Behandlung von 18 mit Natriumdiethylaluminiumhydrid. Es stellte sich heraus, daß $\mathrm{LiAlH}_{4}$ gute Ausbeuten erbringt (bis 70\%) und daß durch Lossungsmittel- und Temperaturvariation das 32: 33-Verhăltnis beeinflußt werden kann. In Tetrahydrofuran erhielten wir bei $-50^{\circ} \mathrm{C}$ weitgehend reines $32(32: 33>10: 1)$, wăhrend bei $66^{\circ} \mathrm{C}$ der Anteil von $3320 \%$ betrug. 
Weniger selektiv verliefen die Reduktionen in Ether: bei - 50 und $35^{\circ} \mathrm{C}$ ermittelten wir 32: 33-Verhăltnisse von 3:1 bzw. 1:1.<smiles>C1C2C3OC4C2C1C34</smiles>

18

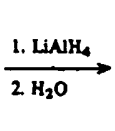<smiles>OC12C3C4C5C3C1C5C42</smiles>

2<smiles>[CH]C1C2C3CC4C1C3C42</smiles>

33<smiles>COC1CC2C3C1C1C2C31</smiles>

Die Umlagerung zu 33 dürfte durch ein Elektrophil ausgelöst werden, das sich an das Sauerstof fatom von $18 \mathrm{zu} 26 \mathrm{a}$ anlagert. Als Kandidaten dafür kommen die Metallionen $\mathrm{Li}^{+}$und $\mathrm{Na}^{+}$oder auch Aluminium- bzw. Borverbindungen in Frage. In Analogie zum protonierten Epoxid 26 laufen dann zwei Konkurrenzreaktionen ab: direkte Öfnung des Dreirings durch Hydrid, was letztlich 32 liefert, und Wagner-Meerwein-Umlagerung zu 12a, das ein Hydridion zu 33a ubernimmt. Dieser letzte Schritt hat in der Darstellung des unsubstituierten Tricylco[2.2.0.0 2.6]hexans (39) aus endo, anti-5,6-Dibrombicyclo[2.1.1]hex-2-en ein Vorbild ${ }^{16 a)}$. Dort wurde gezeigt, daß auf intermediare Kationen, die 12 a vergleichbar sind, das Hydridion jeweils von der endo-Seite her ubbertragen wird ${ }^{46)}$.

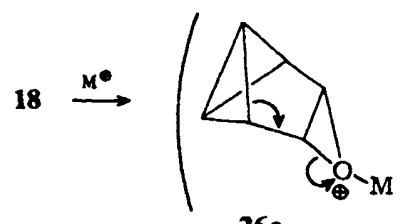

26a

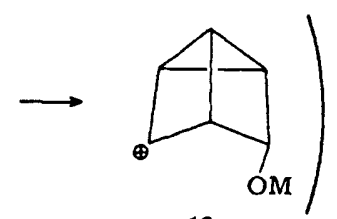

$12 \mathrm{a}$<smiles>[M]OC1C2C3CC4C3C1C42</smiles>

33a

$$
\mathbf{M}=\text { Metall }
$$

Zur vollstăndigen Charakterisierung der Alkohole 32 und 33 haben wir ihr Gemisch mit Natriumhydrid und Methyliodid in das Methylethergemisch $34+35$ umgewandelt und dieses gaschromatographisch getrennt. Die NMR-Spektren lassen keinen Zweifel an der Identităt von 32-35 (Abschnitt F).

In der Literatur ist die Formel 34 nicht neu. Aufgrund der ${ }^{1} H$-NMR-Daten schrieb man diese Konstitution einem der Zersetzungsprodukte des $N$-Nitrosoharnstoffs 36 in Methanol in Gegenwart einer schwachen Base zu ${ }^{47}$ ). Indessen besagen diese Parameter im Vergleich zu unseren Spektren, daß die Autoren nicht 34 sondern 35 in Händen hatten. In gleicher Weise entsteht aus dem Methylderivat 36a nicht 34a sondern 35 a, wie die ${ }^{1} H-N M R-D a t e n$ eindeutig belegen ${ }^{48}$, wenn auch unabhăngig synthetisiertes 35 \& nicht vorliegt. Eine chemische Beweisfuhrung, nămlich die Reduktion mit Lithium in Ethylamin zum unabhängig hergesteltten 37 fuhrte nicht auf die richti-

Chem. Ber. 117 (1984) 
ge Fährte. Es ist bekannt, daß sowohl Tricyclo[2.2.0.0 $0^{2.6}$ hexan $(39)^{49)}$ als auch ein Tricyclo[3.1.0.0 ${ }^{2,6}$ ]hexan-Derivat ${ }^{50)}$ zu Bicyclo[2.1.1] hexan bzw. einem Abkommling davon hydriert werden.<smiles>COC1CC2C3C1C23C</smiles>

34 (34a)

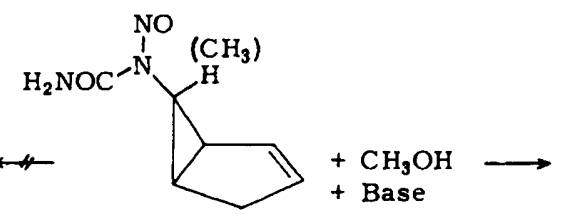

$36(36 a)$<smiles>CC1(C)C2CC3C(O)C1C32</smiles>

$35(35 a)$

37<smiles>COC1CC2CC1C2</smiles>
$\mathrm{A}_{8} \mathrm{ClO}_{4}$<smiles>C[C@H]1CC[C@H]1C</smiles>

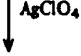<smiles>COC1C2C=CC1C2</smiles>

Als Zwischenstufe der Transformation $36 \rightarrow 35$ kommt das unsubstituierte 6-Bicyclo[2.1.1]hex-2-enyl-Kation in Betracht, das unter kinetischer Kontrolle das Nucleophil an der Zweikohlenstoffbrücke aufnimmt. Genau in dieses Bild paßt die durch Silberperchlorat ausgelöste Umlagerung des angeblichen 34 (d.h. 35) in anti-5-Methoxybicyclo[2.1.1] hex-2-en (38) ${ }^{42 d)}$, wobei das Silberion die Dissoziation von 35 in Methoxid und das 6-Bicyclo[2.1.1]hex-2-enyl-Kation katalysiert und dann die irreversible Rekombination zum thermodynamisch stabileren Produkt eintritt (vgl. die Diskussion zur Bildung von 11 und $28-30$ ). Für das angebliche 34 wurde das Tricyclo[3.1.0.0 2.6] als Vorstufe postuliert ${ }^{47}$. Jedoch existieren Hinweise auf Teilchen dieses Typs nur bei den Additionen von Chlorsulfonylisocyanat ${ }^{40.41)}$ und Tetracyanethylen ${ }^{41}$ an Benzvalen, wo ihr Auftreten innerhalb von Zwitterionen wahrscheinlich ist. Auch diese Befunde deuten auf eine Umlagerung der 3-Tricyclo[3.1.0.0 2.6 hexyl-Kation- in die 6-Bicyclo[2.1.1]hex-2-enyl-Kationeinheit mit extrem niedriger Aktivierungsbarriere hin.

\section{F. Die NMR-Spektren der Tricyclo[3.1.0.0 2,6]- und Tricyclo[2.2.0.0 2,6]hexan- Derivate}

\section{Tricyclo[3.1.0.0 $\left.0^{2.6}\right]$ hexan-Derivate}

Die ${ }^{13} \mathrm{C}$-NMR-Daten dieser Verbindungen (Tab. 2) seien ausgehend von den Parametern des unsubstituierten Kohlenwasserstoffs ${ }^{51}$ ) diskutiert. Damit sind die Absorptionen der Bicyclo[1.1.0]butan-Brückenkopfatome $C$-a und $C$-b durch ihre chemischen Verschiebungen $\delta=0.1-7.2$ und die großen ${ }^{13} \mathrm{C}$-H-Kopplungskonstanten uber eine Bindung $(210-223.5 \mathrm{~Hz})$ charakterisiert. Ebenfalis in einem engen Bereich, nämlich $\delta=31.7-40.5$, mit den typischen direkten Kopp lungskonstanten von 159.9-174.3 $\mathrm{Hz}$ erscheinen die Signale der seitlichen $\mathrm{C}-\mathrm{c}$ und $\mathrm{C}$-d am Bicyclo[1.1.0]butan-System. Die Parameter der Brückenatome $C$-e und $C-f$ werden wie ublich stark 


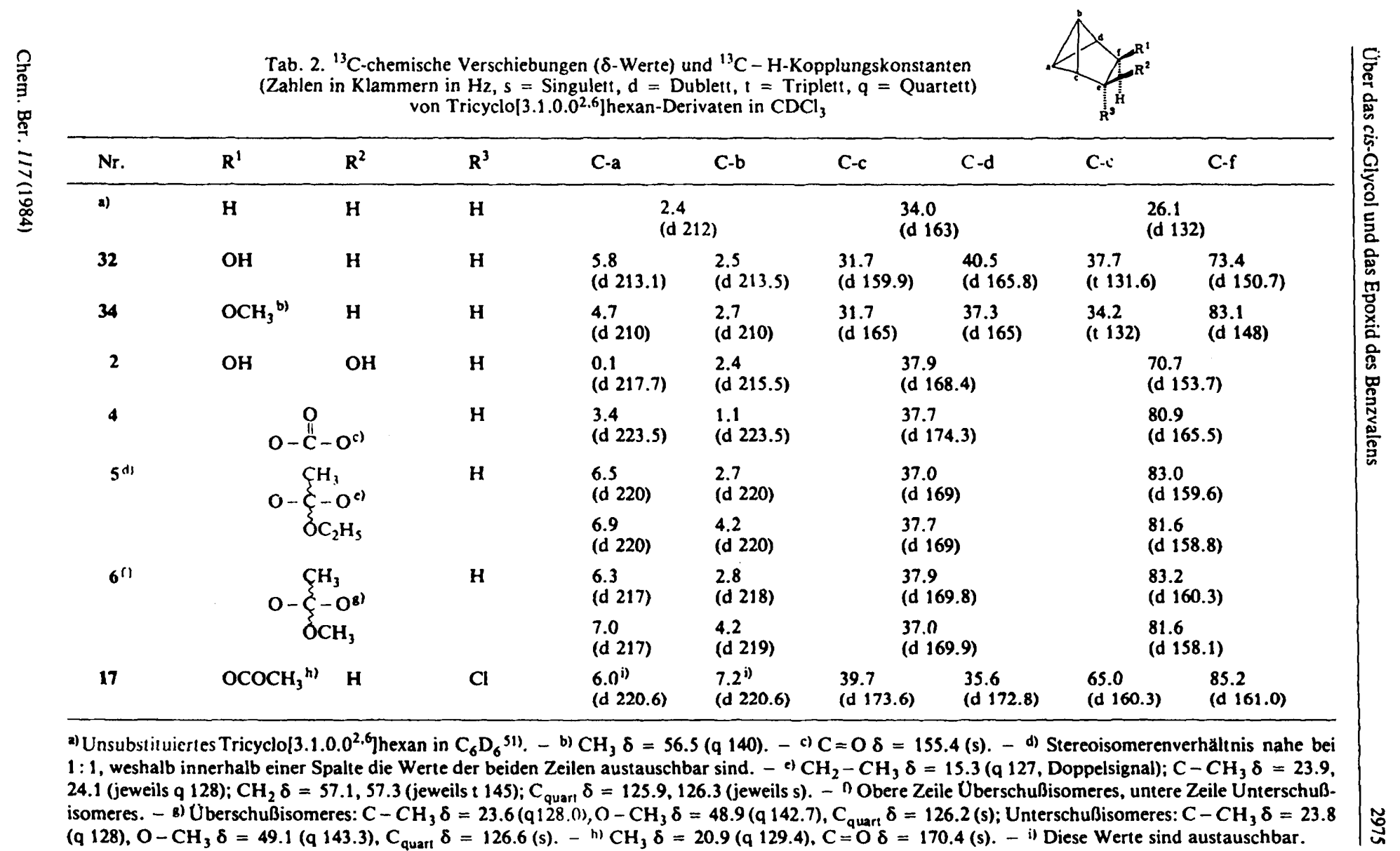


durch die Substituenten beeinflußt. Zur spezifischen Zuordnung dienen bei C-e (zur Unterscheidung von $C$-c und $C-d$ ) in 32 und 34 die Triplettmultiplizităt und in 17 bei $C-e$ und $C-f$ sowie auch bei $C$-c und $C$ - $d$ die in substituierten Cyclohexanen wirksamen Substituenteneffekte ${ }^{27 b)}$. Daß in 2 und 4-6 cis-disubstituierte Tricyclo[3.1.0.0.6.6 3 hexan-Derivate vorliegen, folgt aus zwei Signalen für $\mathrm{C}$-a und $\mathrm{C}$-b, was die $C_{5}$-Symmetrie beweist und so das trans-Glycol mit $C_{2}$-Symmetrie anstelle von 2 ausschließt.

Reichhaltige Informationen bergen die Feinstrukturen der Protonen-gekoppelten Spektren, womit die trans-Stereochemie von 17 beweisbar ist. Die von C-a und C-b herrührenden Signale der cis-disubstituierten Verbindungen 2 und 4-6 weisen einen charakteristischen Feinstrukturunterschied auf, der auch bei Diels-Alder-Addukten des Benzvalens zur Zuordnung herangezogen wurde ${ }^{52)}$. Als Beispiel sei dies am Spektrum von 2 erläutert. Das Signal bei $\delta=0.1$ zeigt neben der 217.7- $\mathrm{Hz}-$ Kopplung noch zwei Aufspaltungen von $1.8 \mathrm{~Hz}$ und eine von $3.7 \mathrm{~Hz}$. Letztere muß aus Symmetriegrunden die geminale Kopplung zum H-Atom des zweiten Bicyclol1.1.0]butanBrückenkopfs sein. Für die 1.8-Hz-Kopplungen konnten dagegen $\mathrm{H}-\mathrm{c}$, d oder $\mathrm{H}-\mathrm{e}, \mathrm{f}$ als Partner fungieren. Jeder Ast des 215.5-Hz-Dubletts des Signals bei $\delta=2.4$ erscheint dagegen als verbrejtertes 8-Hz-Triplett von 3.7-Hz-Dubletts. Letzteres entstammt der zu vorhin analogen geminalen Kopplung, während wir das Triplett der vicinalen Wechselwirkung mit H-e,f zuschreiben. Es ist nämlich kein Grund erkennbar, warum $\mathrm{C}-\mathrm{a}$ und $\mathrm{C}$ - $\mathrm{b}$ nicht in ähnlicher Weise mit $\mathrm{H}-\mathrm{c}, \mathrm{d}$ koppeln soltten, jedoch sind stark unterschiedliche Kopplungskonstanten von $\mathrm{C}-\mathrm{a}$ und $\mathrm{C}-\mathrm{b}$ zu $\mathrm{H}-\mathrm{e}, \mathrm{f}$ zu erwarten, da die Karplus-Conroy-Regel auch für ${ }^{3} J_{\mathrm{C}, \mathrm{H}}$ gültig ist ${ }^{53)}$. Dementsprechend ordnen wir die 8-Hz-Wechselwirkungen den nahezu antiperiplanar angeordneten $\mathrm{C}-\mathrm{b}$ und $\mathrm{H}-\mathrm{e}, \mathrm{f} z \mathrm{z}$, womit die Absorption bei $\delta=2.4 \mathrm{dem}$ zu den $\mathrm{OH}$-Gruppen syn-ständigen $\mathrm{C}$-b angehört. Die synperiplanare Kopplung von $\mathrm{C}$-a beläuft sich auf höchstens $1.8 \mathrm{~Hz}$. Das Phänomen, daß ${ }^{2} J_{C, H}\left(J_{a, H-b}\right.$ $\left.J_{b, H-a}, J_{a, H-c}, J_{b, H-c}\right)$, kleiner ausfallt als ${ }^{3} J_{C, H}\left(J_{b, H-e}\right)$, wird häufig vorgefunden ${ }^{53)}$. Im Spektrum von 4 sind $^{2} J_{a, H \cdot c}$ und ${ }^{2} J_{b, H \cdot c}$ nicht aufgelöst, so daß als Feinstrukturen der C-a- und C-b-Signale ein Dublett bzw. ein Doppeltriplett erscheint (Abb. 1).
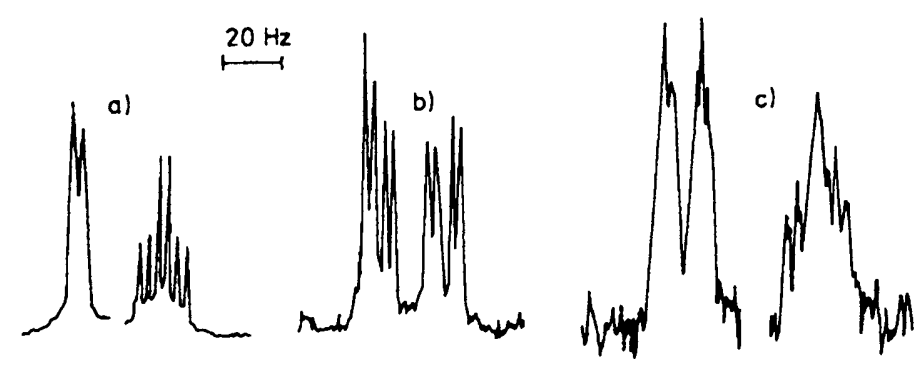

Abb. 1. Protonengekoppelte Signale der Bicyclo[1.1.0]butan-Brückenkopf-Kohlenstoffatome (C-a und C-b in Tab. 2) von a) 4, b) 17, c) 32. Es ist jeweils nur ein Ast des Doppelmultipletts, das aus der direkten $\mathrm{C}$-H-Kopplung resultiert, gezeigt

Obige Argumentation hat zur Konsequenz, daß die Feinstrukturen der Signale von C-a und C-b des trans-Derivats 17 praktisch gleich sein müssen, weil jetzt beide $C$-Atome über ein antiperiplanar-ständiges $\mathrm{H}$-Atom verfugen. Dies trifft $\mathrm{zu}$. $\mathrm{Zu}^{\prime} J_{\mathrm{C}, \mathrm{H}}=220.6 \mathrm{~Hz}$ kommen noch eine 10.3- und eine 3.7- $\mathrm{Hz}$-Aufspaltung $(\delta=6.0) \mathrm{bzw}$. eine von 8.8 und eine von $3.7 \mathrm{~Hz}(\delta=7.2)$. Wie bei 4 sind weitere Kopplungen nicht aufgelost (Abb. 1).

Auch die spezifische Zuordnung der Signale von $C-a$ und $C-b$ in 32 und 34 kann auf dieser Basis getroffen werden. $\mathrm{C}$-a hat nur an $\mathrm{C}$-e ein antiperiplanar-ständiges $\mathrm{H}$-Atom, zu dem eine $12.5-\mathrm{Hz}-$

Chem. Ber. $117(1984)$ 
Kopplung besteht. Dagegen sieht sich C-b zwei derartigen H-Atomen (je einem an C-e und C-f) gegenuber und verursacht daher ein weiter aufgespaltenes $(\approx 3 \mathrm{~Hz}$ ) $8.2-\mathrm{Hz}$-Triplett (Abb. 1).

Aufgrund dieses Kriteriums weicht die Zuordnung der Signale zu C-a und C-b in 2 von denen in den anderen cis-disubstituierten Tricyclo[3.1.0.0.6.6 hexan-Derivaten (4-6) ab. Dort gehort das Hochfeldsignal immer zu C-b. Offenbar üben die OH-Gruppen in 2 einen besonderen Effekt aus, der das $\mathrm{C}$-a-Signal nach hohem Feld verschiebt.

Bemerkenswert ist ferner die Feinstruktur der C-c- und C-d-Signale. Sie besteht in allen Fällen aus einem Doppelmultiplett mit einer großen Kopplung, die recht konstant $12.5 \mathrm{~Hz}$ beträgt und wahrscheinlich auf ${ }^{3} J_{c, H-d}$ bzw. ${ }^{3} J_{d, H-c}$ zurückgeht.

Das ungewöhnliche ${ }^{13} \mathrm{C}$-NMR-Spektrum von 18, in dem der Oxiranring einen Unterschied von $27.6 \mathrm{ppm}$ zwischen den chemischen Verschiebungen von $\mathrm{C}-\mathrm{a}$ und $\mathrm{C}-\mathrm{b}$ verursacht, wurde schon publiziert ${ }^{\text {54). }}$

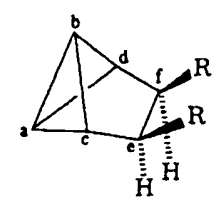

cis: $2-6,18$

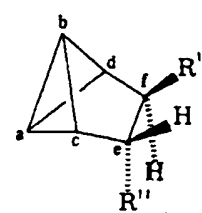

trans: 17,27

Auch die 'H-NMR-Spektren können daruber Auskunft geben, ob ein cis-disubstituiertes Tricyclo/3.1.0.0 2,6]hexan-Derivat vorliegt wie bei $2-6$ und 18 oder ein trans-disubstituiertes, wie bei 17 und 27. Charakteristisch fur letztere ist die kleine Kopplung zwischen $H \cdot e$ und $H \cdot f$, die aus dem ungünstigen Interplanarwinkel von ca. $120^{\circ}$ aufgrund der Karplus-Conroy-Beziehung ${ }^{14 b)}$ resultiert. Sie betrăgt in $341.1 \mathrm{~Hz}$, in 17 höchstens $1 \mathrm{~Hz}$ und ist im Spektrum von 27 und weiteren Beispielen ${ }^{17.40)}$ nicht aufgelost. Wegen der Symmetrie kann $J_{\text {e. }}$ in den cis-disubstituierten Verbindungen nicht ohne weiteres beobachtet werden, jedoch sollte sie wegen des $0^{\circ}$ betragenden Interplanarwinkels von beachtlicher Große sein, was auch $34 \mathrm{mit} 7.0 \mathrm{~Hz}$ stellvertretend bezeugt. Eine genügend gute Auflosung vorausgesetzt, ist die stereochemische Information in den Signalen von $\mathrm{H}$-a und $\mathrm{H}$-b enthaiten. Von diesen Protonen in 3 rührt ein Doppeltriplett bei $\delta=2.08 \mathrm{mit}$ $J_{\mathrm{a}, \mathrm{b}}=7.8 \mathrm{~Hz}$ und $2.2-\mathrm{Hz}$-Kopplungen $\mathrm{zu} \mathrm{H}-\mathrm{c}, \mathrm{d}$ und ein Doppeiquintett bei $\delta=2.53 \mathrm{mit} 1.5-\mathrm{Hz}$ Kopplungen $r u \mathrm{H} \cdot c$,d und $\mathrm{H}$-c.f. Fernkopplungen werden am besten durch $\mathrm{W}$-förmige Bindungs-

Tab. 3. 'H-NMR-Chemische Verschiebungen ( $\delta$-Werte, Diagonalelemente) und Kopplungskonstanten (Absolutwerte in $\mathrm{Hz}$, Nichtdiagonalelemente) von Methyl-(tricyclo[3.1.0.0.6. ether (34) in $\mathrm{CDCl}_{3}$

\begin{tabular}{llllllllll}
\hline & & $1-\mathrm{H}$ & $2-\mathrm{H}$ & $3-\mathrm{H}$ & $4-\mathrm{H}_{\text {cis }}$ & $4-\mathrm{H}_{\text {trans }}$ & $5-\mathrm{H}$ & $6-\mathrm{H}$ \\
\hline & $1-\mathrm{H}$ & 1.88 & 1.7 & a) & 1.7 & 0.7 & 1.7 & 9.3 \\
& $2-\mathrm{H}$ & & 2.28 & 1.6 & a) & a) & 4.7 & 1.5 \\
& $3-\mathrm{H}$ & & & 3.72 & 1.1 & 7.0 & a) & 1.5 \\
& $4-\mathrm{H}_{\text {cis }}$ & & & & 1.14 & 11.6 & 1.3 & 0.7 \\
& $4-\mathrm{H}_{\text {trans }}$ & & & & & 1.62 & 0.8 & 1.5 \\
& $5-\mathrm{H}$ & & & & & & 2.02 & 1.5 \\
& $6-\mathrm{H}$ & & & & & & & 2.10 \\
\hline
\end{tabular}

$\mathrm{CH}_{3}: \delta=3.27$. a) $<0.4 \mathrm{~Hz}$.

Chem. Ber. 117 (1984) 
anordnungen übertragen ${ }^{14 a)}$, und die Bindungen zwischen $\mathrm{H}-\mathrm{e}, \mathrm{f}$ und $\mathrm{H}-\mathrm{b}$ kommen diesem Ideal weit näher als jene zwischen $\mathrm{H}-\mathrm{e}, \mathrm{f}$ und $\mathrm{H}-\mathrm{a}$. Wir weisen daher das die Fernkopplung enthaltende Tieffeldsignal $\mathrm{H}-\mathrm{b} \mathbf{z u}$.

Im 400-MHz-Spektrum von 34 treten keine Bandenüberlappungen auf, so daß alle Kopplungskonstanten, gesichert durch einige Entkopplungsexperimente, nach erster Ordnung ermittelt werden konnten. Tab. 3 offenbart, daß 1-H und 6-H jeweils mit beiden 4-H koppeln, wobei wir aufgrund oben diskutierter Regel die größeren Kopplungen $J_{1,4}$ cis und $J_{4 \text { trans, } 6}$ zuschreiben. Im 7,8,9,10-Tetrachlortetracyclol4.4.0.0 $0^{2,4} \cdot 0^{3.5}$ jeca-7,9-dien hatten wir den ersten derartigen Fall gefunden ${ }^{52)}$. Trotz der all-cis-Anordnung der Bindungen belaufen sich $J_{1,4 \text { trans }}$ und $J_{4 \text {-cis, } 6}$ auf $0.7 \mathrm{~Hz}$, was die Verwandtschaft des gespannten $\sigma$-Systems mit einer $\mathrm{C}=\mathrm{C}$-Doppelbindung unterstreicht. Somit vergleichen sich besagte Fernkopplungen mit Allylwechselwirkungen ${ }^{14 a)}$.

\section{Tricyclo[2.2.0.0 $0^{2.6}$ lhexan-Derivate}

Die ${ }^{13} \mathrm{C}$-NMR-Spektren einer Reihe derartiger Verbindungen wurden gesondert publiziert ${ }^{55)}$, weil endo-ständige Substituenten in 3- und 5-Position außerordentlich große $\gamma$-anti-Effekte ausuben. So absorbiert C-1 von 11 im Vergleich zum Stammkohlenwasserstoff 39 um 27 ppm bei höherem Feld.<smiles>[CH]C1C2C3C1C1C2C31</smiles>

In den 'H-NMR-Spektren sind die Signale von 1- $\mathrm{H}$ und 4-H besonders charakteristisch. $\mathrm{Ob}$ wohl zwei Wege zu 39 bekannt sind ${ }^{16 a, 49)}$, wurde sein Spektrum noch nicht analysiert. Jeweils angenahert ein Quintett von Dubletts verursachen dort besagte Protonen, wobei die wechselseitige Kopplung $J_{1.4}$ mit $0.8 \mathrm{~Hz}$ uberraschend klein ausfallt. Bei 1- $\mathrm{H}\left(\delta=2.94\right.$ in $\left.\mathrm{CDCl}_{3}\right)$ stammt die Quintettmultiplizităt von ăhnlich großen Kopplungen mit $2,6-\mathrm{H}(3.8 \mathrm{~Hz})$ und $3,5-\mathrm{H}_{\text {endo }}(3.0 \mathrm{~Hz}$, W-Anordnung ${ }^{143)}$ beider Bindungspfade), bei $4-H(\delta=2.32)$ von vergleichbar großen Kopplungen mit 3,5- $\mathrm{H}_{\text {exo }}(4.3 \mathrm{~Hz})$ und 2,6- $\mathrm{H}\left(5.0 \mathrm{~Hz}\right.$, W-Anordnung $\left.{ }^{14 \mathrm{a}}\right)$ beider Bindungspfade). Wegen des ungunstigen Interplanarwinkels von ca. $90^{\circ}$ sind $J_{3-e n d o, 4}=J_{4,5-e n d o}$ nicht aufgelöst.

Tab. 4. ${ }^{1} \mathrm{H}$-NMR-Chemische Verschiebungen ( $\delta$-Werte, Diagonalelemente) und Kopplungskonstanten (Absolutwerte in $\mathrm{Hz}$, Nichtdiagonalelemente) von Methyl-(endo-tricyclo[2.2.0.0 2,6]hex-3yl)-ether (35) in $\mathrm{CDCl}_{3}$

\begin{tabular}{lllllllll}
\hline & $1-\mathrm{H}$ & $2-\mathrm{H}$ & $3-\mathrm{H}$ & $4-\mathrm{H}$ & $5-\mathrm{H}_{\text {exo }}$ & $5-\mathrm{H}_{\text {endo }}$ & $6-\mathrm{H}$ \\
\hline & $1-\mathrm{H}$ & 2.55 & 3.5 & a) & 1.0 & a) & 3.3 & 3.5 \\
$2-\mathrm{H}$ & & 2.17 & 2.5 & 4.5 & a) & a) & 4.0 \\
& $3-\mathrm{H}$ & & & 3.96 & 4.2 & a) & a) & a) \\
& $4-\mathrm{H}$ & & & 2.58 & 4.5 & a) & 4.5 \\
& $5-\mathrm{H}_{\text {exo }}$ & & & & 2.44 & 7.3 & 2.5 \\
& $5-\mathrm{H}_{\text {endo }}$ & & & & & 1.80 & a) \\
& $6-\mathrm{H}$ & & & & & & 1.86 \\
\hline
\end{tabular}

$\mathrm{CH}_{3}: \delta=3.21$. a) $<0.4 \mathrm{~Hz}$. 
Aufgrund der endo-Substituenten in 3- und 5-Position von 11;28 und 29 erscheint dort das 1-H-Signal jeweils nur noch als Triplett von Dubletts, wathrend sich an der quint d-Feinstruktur der 4-H-Absorption im Vergleich zu 39 nichts ändert. Das 400-MHz-Spektrum von 35 (Tab. 4) erlaubte die Entnahme der Absolutwerte aller Kopplungskonstanten nach erster Ordnung. Wie die obige Diskussion fordert, verursacht 1-H angenăhert ein Quartett $(\approx 3.4 \mathrm{~Hz})$ von Dubletts $(1.0 \mathrm{~Hz}$ ) und $4-\mathrm{H}$ ein Quintett $(\approx 4.4 \mathrm{~Hz}$ ) von Dubletts.

Unser Dank gilt der Deutschen Forschungsgemeinschaft und dem Fonds der Chemischen Industrie für die Förderung der Arbeit, sowie der Peroxid-Chemie $\mathrm{GmbH}$, Höllriegelskreuth, für eine Spende von 85 proz. Wasserstoffperoxid. Herrn Dr. D. Wendisch, Baye: AG, Leverkusen, danken wir für die Aufnahme der $90.56 \mathrm{MHz}-{ }^{13} \mathrm{C}$ - und $360 \mathrm{MHz}-{ }^{1} \mathrm{H}-\mathrm{NMR}$-Spektren von 22 und die sehr sorgfaltigen Entkopplungsexperimente in den letzteren.

\section{Experimenteller Teil}

'H-NMR: Varian EM 390, Bruker WH 360 und WM 400. - ${ }^{13}$ C-NMR: Bruker WH 90, WH 360 und WM 400. - MS: Varian MAT CH 7. - IR: Beckman AccuLab 4. Schmelzpunkte (nicht korrigiert): Mikroheiztisch der Firma Reichert, Wien.

\footnotetext{
cis-Tricyclo[3.1.0.0.0.6/hexan-3,4-diol (2)
}

a) Oxidation von Benzwalen (1) mit Kaliumpermanganat: Zur Mischung aus $23.2 \mathrm{ml}$ etherischem $1^{56,57)}(1.00 \mathrm{~g}, 12.8 \mathrm{mmol})$ und $50 \mathrm{ml}$ Ethanol tropfte man innerhalb von $10 \mathrm{~min}$ bei -15 bis $-20^{\circ} \mathrm{C}$ eine Losung von $1.00 \mathrm{~g}(25 \mathrm{mmol}) \mathrm{NaOH}$ und $1.80 \mathrm{~g}(11.4 \mathrm{mmol}) \mathrm{KMnO}_{4}$ in $50 \mathrm{ml}$ Wasser, wobei die Farbe nach Braun umschlug. Man filtrierte, entfarbte das Filtrat intit waßßriger $\mathrm{NaHSO}_{3}$-Losung und engte es bei 20 Torr auf ca. $60 \mathrm{ml}$ ein. Nun săttigte man mit $\mathrm{NaCl}$, extrahierte funfmal mit je $20 \mathrm{ml}$ Chloroform, trocknete die vereinigten Extrakte mit $\mathrm{Na}_{2} \mathrm{SO}_{4}$ und engte sie i. Vak. ein. Aus dem Rückstand destillierten bei $70-80^{\circ} \mathrm{C}$ (Bad) $/ 0.01$ Torr $602 \mathrm{mg}(42 \%) 2$ als farblose olige Flüssigkeit, die bei Kuhlung zu Kristallen mit Schmp. von ca. $-5^{\circ} \mathrm{C}$ erstarrte. Steigerung der Ansatzgroße auf $40 \mathrm{mmol}$ senkte die Ausbeute auf $23 \%$ ab. - IR (Film): $3320 \mathrm{~cm}^{-1}$ (breit, OH). - MS (70 eV): $m / e=112\left(10 \%, \mathrm{M}^{+}\right), 83(53), 68(33), 66(100), 65(43), 55(60), 53$ (33), $52(30), 43(43), 40(30), 39(79)$. - ${ }^{1} \mathrm{H}-\mathrm{NMR}\left(\mathrm{CDCl}_{3}\right): \delta=1.90$ (m; 1-H oder $\left.6-\mathrm{H}\right), 2.1-2.4$ (m; 2,5-H und 6-H oder 1-H), 3.87 (br.s; 3,4-H), 4.12 (br.s; OH). - ${ }^{13} \mathrm{C}-\mathrm{NMR}$ : Tab. 1.

$$
\mathrm{C}_{6} \mathrm{H}_{8} \mathrm{O}_{2} \text { (112.1) Ber. C } 64.27 \text { H } 7.20 \text { Gef. C } 64.17 \text { H } 7.32
$$

b) Oxidation von 1 mit tert-Butylhydroperoxid/Osmiumtetroxid: Bei $0^{\circ} \mathrm{C}$ gab man zur Lösung von $20 \mathrm{ml}$ tert-Butylalkohol in $25 \mathrm{ml}$ etherischem $1^{56.57)}(740 \mathrm{mg}, 9.5 \mathrm{mmol}) 0.75 \mathrm{mmol} 20$ proz. wäßriges $\left(\mathrm{C}_{2} \mathrm{H}_{5}\right)_{4} \mathrm{~N}^{+} \mathrm{Cl}^{-}, 2.0 \mathrm{~g} 70$ proz. wäßriges tert-Butylhydroperoxid und schließlich $1.0 \mathrm{ml}$ einer 0.5 proz. Losung von $\mathrm{OsO}_{4}(0.02 \mathrm{mmol})$ in tert-Butylalkohol. Die braune Losung wurde $2 \mathrm{~h}$ bei $0^{\circ} \mathrm{C}$ gerührt und dann $36 \mathrm{~h}$ bei $5^{\circ} \mathrm{C}$ aufbewahrt. Nach Zugabe von $10 \mathrm{ml} 5$ proz. waßßriger $\mathrm{NaHSO}_{3}$-Lơsung engte man bei Raumtemp. i. Vak. ein, extrahierte den braunen Rückstand mit Ether, trocknete die Etherlösung mit $\mathrm{Na}_{2} \mathrm{SO}_{4}$ und engte i. Vak. ein. Der Ruckstand lieferte durch Destillation bei $60-80^{\circ} \mathrm{C}(\mathrm{Bad}) / 0.01$ Torr $580 \mathrm{mg}(54 \%)$ farbloses 2. Hier führte die Vergrößerung des Ansatzes auf $100 \mathrm{mmol}$ nicht zur Ausbeuteminderung.

Bis-p-toluolsulfonsäureester 3: Unter Stickstoff gab man zur gerührten Lösung von $1.00 \mathrm{~g}$ $(8.92 \mathrm{mmol}) 2$ in $20 \mathrm{ml}$ absol. Pyridin bei $0^{\circ} \mathrm{C}$ portionsweise $3.82 \mathrm{~g}(20.0 \mathrm{mmol}) p$-Toluolsulfochlorid. Man rührte $5 \mathrm{~h}$ bei $0^{\circ} \mathrm{C}$, goß dann in $50 \mathrm{ml}$ Eiswasser und filtrierte die ausgefallenen farblosen Kristalle ab: $2.59 \mathrm{~g}(69 \%) 3$, Schmp. nach Umlösen aus Methanol $103-104^{\circ} \mathrm{C}$. - MS $(70$ eV): $m / e=249\left(8 \%, \mathrm{M}^{+}-\mathrm{CH}_{3} \mathrm{C}_{6} \mathrm{H}_{4} \mathrm{SO}_{3}\right), 155$ (30), 91 (100), 65 (27), 39 (14). - ${ }^{1} \mathrm{H}-\mathrm{NMR}$

Chem. Ber. 117(1984) 
$\left(\mathrm{CDCl}_{3}\right): \delta=2.08\left(\mathrm{dt}, J_{1,6}=7.8 \mathrm{~Hz}, J_{1.2}=2.2 \mathrm{~Hz} ; 1-\mathrm{H}\right), 2.30$ (br.s; 2,5-H), $2.44\left(\mathrm{~s}, \mathrm{CH}_{3}\right), 2.53$ (dquint, $J_{2,6}=J_{3,6}=1.5 \mathrm{~Hz} ; 6-\mathrm{H}$ ), 4.64 (br.s, 3,4-H), 7.31 und 7.81 (AA'BB'-Spektrum, $\mathrm{C}_{6} \mathrm{H}_{4}$ ).

$\mathrm{C}_{20} \mathrm{H}_{20} \mathrm{O}_{6} \mathrm{~S}_{2}$ (420.5) Ber. C 57.13 H $4.79 \mathrm{~S} 15.25$ Gef. C 56.83 H $4.81 \mathrm{~S} 15.21$

7,9-Dioxatetracyclo/4.3.0.0.2.4. $0^{3,5}$ Inonan-8-on (4): Unter Stickstoff tropfte man zur gerührten Losung von $600 \mathrm{mg}(5.35 \mathrm{mmol}) 2 \mathrm{in} 25 \mathrm{ml}$ absol. Pyridin bei $0^{\circ} \mathrm{C} 1 \mathrm{ml}$ Phosgen, wobei sich sofort ein farbloser Niederschlag bildete. Man ruhrte $30 \mathrm{~min}$ bei $0^{\circ} \mathrm{C}$, hydrolysierte mit $10 \mathrm{ml} \mathrm{Was}-$ ser und extrahierte dreimal mit je $30 \mathrm{ml}$ Ether. Nach Trocknen der Etherlosung mit $\mathrm{Na}_{2} \mathrm{SO}_{4}$ und Einengen bei 20 Torr hinterblieben $650 \mathrm{mg}(88 \%) 4$ in farblosen Kristallen. Umlosen aus Methanol erbrachte $530 \mathrm{mg}(72 \%)$ mit Schmp. $76-77^{\circ} \mathrm{C}$. - IR $(\mathrm{KBr}): 1765 \mathrm{~cm}^{-1}(\mathrm{C}=0)$. - MS (70 eV): $m / e=138\left(8 \%, \mathrm{M}^{+}\right), 66(100), 65(48), 40(45), 39(56) .-{ }^{1} \mathrm{H}-\mathrm{NMR}\left(\mathrm{CDCl}_{3}\right): \delta=2.36(\mathrm{dt}$, $\left.J_{3.4}=8.3 \mathrm{~Hz}, J_{2,3}=1.9 \mathrm{~Hz} ; 3-\mathrm{H}\right), 2.51$ (d von $\left.\mathrm{m} ; 4-\mathrm{H}\right), 2.65$ (br.s; $\left.2,5-\mathrm{H}\right), 4.85$ (br.s; $\left.1,6-\mathrm{H}\right)$. ${ }^{13}$ C-NMR: Tab. 1 .

$$
\mathrm{C}_{7} \mathrm{H}_{6} \mathrm{O}_{3} \text { (138.1) Ber. } \mathrm{C} 60.87 \mathrm{H} 4.38 \text { Gef. } \mathrm{C} 60.56 \mathrm{H} 4.12
$$

8-Ethoxy-8-methyl-7,9-dioxatetracyclo/4.3.0.0.0.4. $0^{3,5}$ /nonane $5 \mathrm{a}$ und $5 \mathrm{~b}$ : Aus der Losung von $1.42 \mathrm{~g}(12.7 \mathrm{mmol}) 2,5 \mathrm{ml}(27.3 \mathrm{mmol}) 1,1,1-$ Triethoxyethan und $161 \mathrm{mg}(1.32 \mathrm{mmol})$ Benzoesăure in $120 \mathrm{ml}$ absol. Benzol destillierte man bei Normaldruck innerhalb von $6 \mathrm{~h} 40 \mathrm{ml}$ Losungsmittel ab. Nach Abkuhlen auf Raumtemp. gab man $1.00 \mathrm{~g} \mathrm{Na} \mathrm{CO}_{3} \mathrm{zu}$, filtrierte und engte i. Vak. ein. Aus dem Ruckstand $\left(1.89 \mathrm{~g}, 82 \%\right.$ ) destillierten bei $30-35^{\circ} \mathrm{C}$ (Bad) $/ 0.01$ Torr $1.13 \mathrm{~g}(49 \%) 5 \mathrm{a}$ und $5 \mathrm{~b}$ als gelbliche Flussigkeit, aufgrund des ${ }^{1} \mathrm{H}$-NMR-Spektrums ein 52:48-Gemisch. - MS $(70 \mathrm{eV}): m / e=167\left(2 \%, \mathrm{M}^{+}-\mathrm{CH}_{3}\right), 137\left(35, \mathrm{M}^{+}-\mathrm{OC}_{2} \mathrm{H}_{9}\right), 105(19), 95(28), 77(36), 68$ (20), 66 (96), 65 (40), 61 (49), 45 (26), 43 (100), 40 (28), 39 (46). - ${ }^{1} \mathrm{H}-\mathrm{NMR}\left(\mathrm{CDCl}_{3}\right)$ : Uberschußisomeres: $\delta=1.15\left(\mathrm{t}, J=7.2 \mathrm{~Hz} ; \mathrm{CH}_{2}-\mathrm{CH}_{3}\right), 1.60\left(\mathrm{~s}, \mathrm{C}_{\text {quart }}-\mathrm{CH}_{3}\right), 1.8-2.5(\mathrm{~m} ; 2,5-\mathrm{H}, 3-\mathrm{H}$, 4-H), 3.45 (q; $\mathrm{CH}_{2}$ ), 4.56 (s; $1,6-\mathrm{H}$ ). Unterschußisomeres: $\delta=1.20$ (t, $J=7.2 \mathrm{~Hz} ; \mathrm{CH}_{2}-\mathrm{CH}_{3}$ ), 1.43 (s; $\left.\mathrm{C}_{\text {quast }}-\mathrm{CH}_{3}\right), 1.8-2.5(\mathrm{~m} ; 2,5-\mathrm{H}, 3-\mathrm{H}, 4-\mathrm{H}), 3.78\left(\mathrm{q} ; \mathrm{CH}_{2}\right), 4.43(\mathrm{~s} ; 1,6-\mathrm{H})$. ${ }^{13} \mathrm{C}-\mathrm{NMR}:$ Tab. 1.

$$
\mathrm{C}_{10} \mathrm{H}_{14} \mathrm{O}_{3} \text { (182.2) Ber. C } 65.92 \text { H } 7.74 \text { Gef. C } 65.81 \text { H } 8.13
$$

8-Methoxy-8-methyl-7,9-dioxatetracyclo/4.3.0.0.4. $0^{3.5}$ Jnonane $6 \mathrm{a}$ und $6 \mathrm{~b}$ : Gemaß der Vorschrift fur $5 \mathrm{a}$ und $5 \mathrm{~b}$ gewann man aus $500 \mathrm{mg}(4.46 \mathrm{mmol}) 2650 \mathrm{mg}(87 \%) 6 \mathrm{a}$ und $6 \mathrm{~b}$ als farbloses flussiges 72:28-Gemisch mit Sdp. $25-35^{\circ} \mathrm{C}(\mathrm{Bad}) / 0.01$ Torr. $-\mathrm{MS}(70 \mathrm{eV}): \mathrm{m} / \mathrm{e}=168$ $\left(0.4 \%, \mathrm{M}^{+}\right), 153\left(3, \mathrm{M}^{+}-\mathrm{CH}_{3}\right), 137\left(44, \mathrm{M}^{+}-\mathrm{OCH}_{3}\right), 136(45), 105(16), 78(12), 77(32), 66$ (100), 65 (43), $43(83), 40(24), 39(33)$. $-{ }^{1} \mathrm{H}-\mathrm{NMR}\left(\mathrm{CDCl}_{3}\right)$ : Uberschußisomeres: $\delta=1.56(\mathrm{~s}$; $\left.\mathrm{CCH}_{3}\right), 1.9-2.5(\mathrm{~m} ; 2,5-\mathrm{H}, 3-\mathrm{H}, 4-\mathrm{H}), 3.14\left(\mathrm{~s} ; \mathrm{OCH}_{3}\right), 4.57$ (br.s; 1,6-H). Unterschußisomeres: $\delta=1.37\left(\mathrm{~s} ; \mathrm{CCH}_{3}\right), 1.9-2.5(\mathrm{~m} ; 2,5-\mathrm{H}, 3-\mathrm{H}, 4-\mathrm{H}), 3.38\left(\mathrm{~s} ; \mathrm{OCH}_{3}\right), 4.45(\mathrm{br} . \mathrm{s} ; 1,6-\mathrm{H})$. ${ }^{13}$ C-NMR: Tab. 1.

$$
\mathrm{C}_{9} \mathrm{H}_{12} \mathrm{O}_{3} \text { (168.2) Ber. C } 64.27 \text { H } 7.19 \text { Gef. } \mathrm{C} 63.32 \text { H } 7.33
$$

endo-5-(Phenylthio)bicyclo/2.1.1/hexan-cis-2,3-diol (8): $300 \mathrm{mg}$ (2.68 mmol) 2 und $295 \mathrm{mg}$ (2.68 mmol) Thiophenol wurden in $10 \mathrm{ml}$ Benzol 30 min lang mit 350-nm-Licht bestrahlt (Grăntzel-Reaktor 400), wobei sich 8 in farblosen glänzenden Blăttchen abschied. Filtration und Trocknen erbrachte $400 \mathrm{mg}(67 \%)$, Schmp. $134-136^{\circ} \mathrm{C}$. Die Analysenprobe wurde durch Auflosen in Methylenchlorid, Abfiltrieren vom Ungelösten und Ausfallen mit Pentan bereitet. - IR (KBr): 3230, 3425 (beide breit, $\mathrm{OH}), 159 \mathrm{~cm}^{-1}(\mathrm{C}=\mathrm{C})$. $-\mathrm{MS}(70 \mathrm{eV}): m / e=222\left(13 \%, \mathrm{M}^{+}\right)$, 165 (100), 147 (20), $110(51), 87$ (17), 78 (11), 77 (13), 67 (29), 66 (12), 65 (13), 51 (11), 41 (15), 39 (17). - 'H-NMR ([D $]$ Aceton): $\delta=1.47\left(\mathrm{dm}, J_{6 s y n, 6 a n t i}=7.8 \mathrm{~Hz} ; 6-\mathrm{H}_{\text {anti }}\right), 1.80\left(\mathrm{~d} ; 6-\mathrm{H}_{\text {syn }}\right)$, $2.65(\mathrm{~m} ; 1,4-\mathrm{H}), 3.46\left(\mathrm{t}, J_{1,5}=2.7 \mathrm{~Hz} ; 5-\mathrm{H}\right), 4.07$ (br.s; OH), 4.39 (br.s; 3.4-H), $7.1-7.4$ (m; $\mathrm{C}_{6} \mathrm{H}_{5}$ ).

$\mathrm{C}_{12} \mathrm{H}_{14} \mathrm{SO}_{2}$ (222.3) Ber. C 64.83 H $6.35 \mathrm{~S} 14.43$ Gef. C 64.60 H $6.33 \mathrm{~S} 14.45$ 
Eine 100-mg-Probe von 2 in $0.5 \mathrm{ml} \mathrm{CDCl}{ }_{3}$ blieb auf Zusatz einer aquivalenten Menge Thiophenol bei Raumtemp. unverăndert. Auch die Einwirkung von Sonnenlicht loste keine Reaktion aus, und erst $350-\mathrm{nm}$-Licht fuhrte in $30 \mathrm{~min}$ zur quantitativen Umsetzung.

endo,endo-Tricyclo[2.2.0.0.0.6 Jhexan-3,5-diol (11): Die Lösung von $500 \mathrm{mg}$ (1.19 mmol) 3 in $10 \mathrm{ml} 60$ proz. wäßrigem Aceton und $500 \mathrm{mg}(4.34 \mathrm{mmol}) \mathrm{N}, \mathrm{N}$-Diisopropylethylamin (oder der aquiv. Menge Triethylamin) wurde $90 \mathrm{~min}$ auf $75^{\circ} \mathrm{C}$ erhitzt und dann i. Vak. eingeengt. Ein ${ }^{\prime} \mathrm{H}$ NMR-Spektrum des braunen, teilkristallinen Ruckstands zeigte die vollständige Umsetzung von 3 an. Aus diesem Rückstand destillierten bei $50-60^{\circ} \mathrm{C}(\mathrm{Bad}) / 0.01$ Torr $51 \mathrm{mg}(38 \%) 11$ als gelbliche Flüssigkeit, die beim Abkuhlen kristallisierte und sich schon bei leichtem Erwarmen dunket färbte. Die Labilităt verhinderte eine korrekte Elementaranalyse. - IR (Film): $3310 \mathrm{~cm}^{-1}$ (breit, $\mathrm{OH}) .-\mathrm{MS}(70 \mathrm{eV}): m / e=112\left(1 \%, \mathrm{M}^{+}\right), 95(37), 94(29), 78(38), 7 i(52), 66(100), 65(59), 44$ (37), 42 (44), 41 (47), $40(35), 39(87)$. $-{ }^{1} \mathrm{H}-\mathrm{NMR}\left(\mathrm{CDCl}_{3}\right): \delta=2.11$ (td, $J_{1,2}=3.7 \mathrm{~Hz}, J_{1,4}=$ $1.0 \mathrm{~Hz} ; 1-\mathrm{H}$ ), $2.33(\mathrm{~m} ; 2.6-\mathrm{H}), 2.81$ (quint d, $\left.J_{2.4}=J_{3.4}=4.2 \mathrm{~Hz} ; 4-\mathrm{H}\right), 4.41(\mathrm{~m} ; 3.5-\mathrm{H}), 4.33$ (br.; OH). - ${ }^{13} \mathrm{C}$-NMR: Lit. ${ }^{59)}$.

$$
\mathrm{C}_{6} \mathrm{H}_{8} \mathrm{O}_{2} \text { (112.1) Ber. C } 64.27 \mathrm{H} 7.19 \text { Gef. C } 65.66 \mathrm{H} 7.14
$$

trans-3-Acetoxy-4-chlortricyclo/3.1.0.0 $0^{2.6}$ hexan (17) und endo-5-Acetoxy-anti-6-chlorbicyclo12.1.1]hex-2-en (16): $910 \mathrm{mg}$ (3.26 mmol) Chlortriphenylmethan wurden im Ölpumpenvak. vom Thionylchlorid befreit, das zur Stabilisierung zugesetzt worden war. Unter Stickstoff gab man $548 \mathrm{mg}$ ( $3.26 \mathrm{mmol}$ ) 6a/6 b in $2 \mathrm{ml}$ Dichlormethan zu, kochte $90 \mathrm{~min}$ unter Rückfluß und entfernte das Solvens i. Vak. Aus dem Rückstand destillierten bei $45-50^{\circ} \mathrm{C}$ (Bad) $/ 0.01$ Torr $320 \mathrm{mg}$ $(56 \%)$ einer farblosen Flüssigkeit, die neben 17 und 16 im Verhältnis $95: 5$ ('H-NMR) geringfügige Verunreinigungen enthielt (Banden bei $\delta=7.3-8.2$ ), deren Abtrennung nicht gelang.

16: ${ }^{1} \mathrm{H}$-NMR $\left(\mathrm{CDCl}_{3}\right): \delta=2.1\left(\mathrm{CH}_{3}\right.$, verdeckt durch $\mathrm{CH}_{3}$-Signal von 17), 3.12 (pseudo q mit Linienabstand $2.2 \mathrm{~Hz} ; 1,4-\mathrm{H}), 4.21(\mathrm{~s} ; 6-\mathrm{H}), 5.97\left(\mathrm{t}, J_{1.5}=2.2 \mathrm{~Hz} ; 5-\mathrm{H}\right), 6.59$ (pseudo $\mathrm{t} ; 2,3-\mathrm{H}$ ).

17: IR (Film): $1738 \mathrm{~cm}^{-1}(C=O)$. - MS $(70 \mathrm{eV}): m / e=174,172\left(0.1 \%, 0.3 \%, \mathrm{M}^{+}\right), 112(29)$, 95 (54), 94 (22), 77 (46), $66(36), 43(100), 39(20)$. - ' $\mathrm{H}-\mathrm{NMR}\left(\mathrm{CDCl}_{3}\right): \delta=2.06\left(\mathrm{~s} ; \mathrm{CH}_{3}\right), 2.38$ und 2.46 (jeweils br.s, jeweils $2 \mathrm{H}$ ), $3.48(4-\mathrm{H})$ und $4.86(3-\mathrm{H})$ (jeweils pseudo $\mathrm{q}$ mit Linienabstand ca. $1 \mathrm{~Hz}$ ). - ${ }^{13} \mathrm{C}$-NMR: Tab. 1.

$$
\left.\begin{array}{lrl}
\mathrm{C}_{8} \mathrm{H}_{9} \mathrm{ClO}_{2} \text { (172.6) } & \text { Ber. C } 55.67 \text { H } 5.26 \\
& \text { Gef. C } 54.70 \text { H } 5.32 \\
& \text { C } 56.57 \text { H } 5.55
\end{array}\right\} \begin{aligned}
& \text { Substanzen aus ver- } \\
& \text { schiedenen Ansätzen }
\end{aligned}
$$

\section{7-Oxatetracyclo[4.1.0.0.4. $.0^{3.5}$ Jheptan (Benzwalenoxid) (18)}

a) Aus dem trans-Chlorhydrinacetat 17: Unter Stickstoff tropfte man bei $0^{\circ} \mathrm{C} 660 \mathrm{mg}(3.82$ mmol) 17 in $20 \mathrm{ml}$ absol. Triglyme zu $6.50 \mathrm{~g}(120 \mathrm{mmol})$ frisch hergestelltem $\mathrm{NaOCH}_{3}$ in $120 \mathrm{ml}$ absol. Triglyme. Man ruhrte $15 \mathrm{~h}$ bei $0^{\circ} \mathrm{C}$, filtrierte unter Stickstoff den Niederschlag ab und kondensierte aus dem Filtrat unter leichtem Erwärmen $\left(<40^{\circ} \mathrm{C}\right)$ die flüchtigen Komponenten bei 0.01 Torr in eine auf $-78^{\circ} \mathrm{C}$ gekuhlte Vorlage. Aus dem Kondensat verfluchtigte sich bei Raumtemp. und 15 Torr Essigsäure-methylester ( $\left.{ }^{\prime} \mathrm{H}-\mathrm{NMR}\right)$ und bei $30^{\circ} \mathrm{C}$ und 0.01 Torr eine bei $-78^{\circ} \mathrm{C}$ aufgefangene Fraktion, die neben $180 \mathrm{mg} 18(50 \%)$ kleine Mengen an Mono- und Diglyme (Verunreinigungen des Triglyme) enthielt. Der Nachweis von 18 beruht auf dem Vergleich ('H-NMR) mit dem Produkt aus b).

b) Aus Benzualen (1) durch Epoxidierung: Bei $0^{\circ} \mathrm{C}$ tropfte man unter Stickstoff und unter Ruhren zu $15 \mathrm{ml}$ etherischem $1^{56.57)}$ (450 mg, $5.76 \mathrm{mmol}$ ), in dem ca. 1 g K ${ }_{2} \mathrm{CO}_{3}$ suspendiert war, innerhalb von $15 \mathrm{~min} 1.88 \mathrm{~g}$ (10.4 mmol) $N$-Benzoylpercarbamidsäure ${ }^{23)}$ als $0.3-0.5 \mathrm{M}$ Lossung in absol. Tetrahydrofuran (THF). Unter weiterem Rühren ließ man auf Raumtemp. kommen. Es entwickelte sich ein Gas, ein Niederschlag schied sich ab, und die Lösung verfärbte sich braun. Nach $15 \mathrm{~h}$ filtrierte man, engte das Filtrat bei $0^{\circ} \mathrm{C}$ und 15 Torr ein und kondensierte aus dem 
Rückstand unter Erwărmen auf $50^{\circ} \mathrm{C} \mathrm{im} 10^{-3}$-Torr-Vak. die fluchtigen Komponenten in eine auf $-78^{\circ} \mathrm{C}$ gekuhlte Vorlage. Die so erhaltene Flussigkeit bestand hauptsăchlich aus 18 und THF. Letzteres wurde weitgehend bei $0^{\circ} \mathrm{C}$ im 15-Torr-Vak. abgetrennt, und aus dem Ruckstand destillierten bei $40-70^{\circ} \mathrm{C}(\mathrm{Bad}) / 14$ Torr $320 \mathrm{mg}$ einer farblosen viskosen Flussigkeit, die aufgrund der NMR-Analyse ca. $290 \mathrm{mg}(54 \%) 18$ und als Hauptverunreinigung THF enthielt. Wegen der hohen Fluchtigkeit von 18 ging die weitere Anreicherung mit großen Substanzverlusten einher. Korrekte Elementaranalysenwerte wurden nicht erhalten, da die Abtrennung einer Verunreinigung, die aufgrund ihrer NMR-Banden vermutlich aus THF und Persăure entstanden war, mißlang. Die Ansatzgroße wurde ohne Ausbeuteverluste auf $64 \mathrm{mmol} 1$ gesteigert. - IR (Film): $3110(w), 3050$ $(\mathrm{m}), 3000(\mathrm{w}), 1400(\mathrm{w}), 1350(\mathrm{~m}), 1288(\mathrm{~m}), 1143(\mathrm{~m}), 1100(\mathrm{~s}), 977(\mathrm{~m}), 884(\mathrm{~s}), 852(\mathrm{~s}), 762(\mathrm{~s})$, $740(\mathrm{~s}), 660(\mathrm{~s}) \mathrm{cm}^{-1}$. - MS $(70 \mathrm{eV}): m / e=94\left(17 \%, \mathrm{M}^{+}\right), 78(20), 77(37), 68(37), 66(96), 65$ (76), 51 (26), 50 (19), $40(51), 39(100)$. - ' $\mathrm{H}-\mathrm{NMR}\left(\mathrm{CDCl}_{3}\right): \delta=2.19\left(\mathrm{dt}, J_{3,4}=8.1 \mathrm{~Hz}, J_{2,4}=\right.$ $2.6 \mathrm{~Hz} ; 4-\mathrm{H}), 2.33(\mathrm{~m} ; 2,5-\mathrm{H}), 2.98\left(\mathrm{dt}, J_{2,3}=1.1 \mathrm{~Hz} ; 3-\mathrm{H}\right), 3.41$ (br.s; $\left.1,6-\mathrm{H}\right) .-{ }^{13} \mathrm{C}-\mathrm{NMR}$ : Lit. ${ }^{\text {s4). }}$.

$\left[3,4-D_{2}\right]-7-$ Oxatetracyclo[4 $1.0 .0^{2.4} \cdot 0^{3.5}$ Jheptan (18a) wurde auf zwei Wegen hergestellt. Auf Weg 1 epoxidierten wir $\left(1,6-D_{2}\right]$ Benzvalen ${ }^{16 a)}$ wie unter Vorschrift b). Weg 2 geht von 18 aus, das mit Kalium-tert-butoxid in $\left[D_{6}\right]$ Dimethylsulfoxid dem H/D-Austausch unterworfen wurde. Allerdings gelang es dabei nicht, das Solvens vollstandig abzutrennen, was beim Versuch der Thermolyse dazu fuhrte, daß sich $18 \mathrm{a}$ nicht in $19 \mathrm{a}$ umwandelte, sondern sich rasch in unbekannter Weise zersetzte. - Das ${ }^{1} \mathrm{H}-\mathrm{NMR}$-Spektrum von $18 \mathrm{a}$ zeigt in $\mathrm{CDCl}_{3}$ je ein br.s bei $\delta=2.33$ und 3.41.

Thermolysen von 18

a) Bei niedriger Konzentration: Ca. $100 \mathrm{mg} 18 \mathrm{mit}$ Zusätzen an Cyclohexan als Locksubstanz für die ${ }^{1} \mathrm{H}$-NMR-Spektroskopie und Tetramethylethylendiamin zur Abpufferung evtl. vorhandener Säurespuren und Resten von THF und Chloroform, letzteres als Folge unserer früheren Aufarbeitungsprozedur bei der Bereitung von 18, wurden zusammen mit $0.5 \mathrm{ml} \mathrm{C}_{6} \mathrm{D}_{6}$ bei $10^{-4}$ Torr in ein NMR-Rohr kondensiert. Nach dem Abschmelzen unter Vakuum wurde in einem Trockenschrank erhitzt, wobei erst bei $145^{\circ} \mathrm{C}$ eine Reaktion eintrat. Man verfolgte den Verlauf der Thermolyse mit Hilfe von ${ }^{1} \mathrm{H}-\mathrm{NMR}$-Spektren. Nach $11 \mathrm{~h}$ war 18 nicht mehr nachweisbar. Neue Signale starker Intensităt waren feldaufwärts von $\delta=4.6$ beobachtbar. Daneben waren mit wenigen Prozent Ausbeute 19 und 20/21 im Verhältnis 7:3 entstanden (Ausbeuteabschätzung durch Vergleich mit dem $\mathrm{C}_{6} \mathrm{D}_{5} \mathrm{H}$-Signal). Bei 0.01 Torr und Raumtemp. wurden die flüchtigen Anteile in eine auf $-78^{\circ} \mathrm{C}$ gekühlte Falle kondensiert. Das NMR-Spektrum des Kondensats bewies das Vorliegen von 19 und $20 / 21$.

19: ${ }^{1} \mathrm{H}$-NMR: Tab. 1. - ${ }^{13} \mathrm{C}-\mathrm{NMR}\left(\mathrm{C}_{6} \mathrm{D}_{6}\right): \delta=51.7$ (C-5), 82.6 (C-1) 105.0 (C-4), 135.5, 148.8, 149.0 .

20/21: ' $\mathrm{H}$-NMR $\left(\mathrm{C}_{6} \mathrm{D}_{6}\right): \delta=5.08\left(\mathrm{~m}, \mathrm{H}_{\alpha}\right), 5.48\left(\mathrm{~m}, \mathrm{H}_{\beta}\right), 5.95\left(\mathrm{~m}, \mathrm{H}_{\gamma}\right)$; die Bandenform stimmt mit der Abbildung in Lit. ${ }^{29)}$ überein; Differenzen in den chemischen Verschiebungen gehen auf das unterschiedliche Lösungsmittel zurük.

Aus dem Rückstand der Umkondensation destillierten bei $110-120^{\circ} \mathrm{C}(\mathrm{Bad}) / 0.01$ Torr $10 \mathrm{mg}$ eines gelben Öls. $-{ }^{1} \mathrm{H}-\mathrm{NMR}\left(\mathrm{CDCl}_{3}\right): \delta=2.1-2.6(\mathrm{~m}$; rel. Intensităt $9 \mathrm{H}), 2.8-3.1(\mathrm{~m} ; 2 \mathrm{H})$, $3.87\left(\mathrm{~d}, J=8.1 \mathrm{~Hz} ; 1 \mathrm{H}\right.$; da dieses Signal beim Schütteln mit $\mathrm{D}_{2} \mathrm{O}$ verschwindet, handelt es sich um eine OH-Bande), $4.1-4.5(\mathrm{~m} ; 4 \mathrm{H}) .-{ }^{13} \mathrm{C}-\mathrm{NMR}\left(\mathrm{CDCl}_{3}\right)$ : 5 Banden bei $\delta=10.5-12.5,12$ Banden bei 24.4-28.5, 7 Banden bei 48.0-49.2, 13 Banden bei 69.0-77.6.

b) Bei hoher Konzentration: $811 \mathrm{mg}(8.64 \mathrm{mmol}) 18$, nur mit Resten von THF und $\mathrm{CHCl}_{3}$ verunreinigt, wurden mit $0.35 \mathrm{ml} \mathrm{C}_{6} \mathrm{D}_{6}$ in ein NMR-Rohr kondensiert. Nach dem Abschmelzen thermolysierte man $11 \mathrm{~h}$ bei $145^{\circ} \mathrm{C}$ und identifizierte nun $349 \mathrm{mg} \mathrm{(43 \% )} 19$ durch NMR-Analyse. Die fluchtigen Anteile wurden im 0.01-Torr-Vak. entfernt. Beim Behandeln des Ruckstandes mit Ben- 
zol schieden sich $176 \mathrm{mg}$ (22\%) 4,11-Dioxapentacyclo[7.3.2.0.0.8. (22) in gelblichen Kristallen ab, die bei $40^{\circ} \mathrm{C}$ (Bad) $/ 10^{-3}$ Torr sublimierten; Schmp. der jetzt farblosen Kristalle $124-125^{\circ} \mathrm{C}$. Das restliche Material stimmte mit dem Rückstand von Versuch a) überein. - 22: IR (KBr): $3092(\mathrm{w}), 3041$ (w), $3025(\mathrm{w}), 3010(\mathrm{~m}), 2942(\mathrm{~m}), 2930(\mathrm{~m}), 2915(\mathrm{~m})$, $1605(C=C, m), 1396(w), 1378(w), 1309(m), 1278(w), 1258(w), 1247(w), 1236(m), 1215(w)$, $1188(\mathrm{~m}), 1131(\mathrm{~s}), 1123(\mathrm{~m}), 1098(\mathrm{w}), 1058(\mathrm{~m}), 1041(\mathrm{~s}), 1030(\mathrm{~m}), 1008(\mathrm{~m}), 982(\mathrm{~m}), 957(\mathrm{~m})$ $948(\mathrm{~m}), 933(\mathrm{~m}), 903(\mathrm{~s}), 891(\mathrm{~m}), 850(\mathrm{w}), 838(\mathrm{~m}), 827(\mathrm{w}), 810(\mathrm{w}), 738(\mathrm{~s}), 722(\mathrm{~m}), 707(\mathrm{~m})$ $\mathrm{cm}^{-1}$. $-\mathrm{MS}(70 \mathrm{eV}): m / e=188\left(2 \%, \mathrm{M}^{+}\right), 120(11), 92(13), 91(100), 65(11), 39(12) .-{ }^{1} \mathrm{H}-$ NMR $\left(\mathrm{CDCl}_{3}, 360 \mathrm{MHz}\right): \delta=2.32\left(\mathrm{dt}, J_{2,8}=8.5 \mathrm{~Hz}, J_{7,8}=J_{8,9}=3.5 \mathrm{~Hz} ; 8-\mathrm{H}\right), 2.59(\mathrm{~m}$, $\left.J_{1,2} \approx 4 \mathrm{~Hz}, J_{2,3}=2.8 \mathrm{~Hz}, J_{2,7}=1.5 \mathrm{~Hz} ; 2-\mathrm{H}\right), 2.89\left(\mathrm{~m}, J_{3,7}=7.5 \mathrm{~Hz}, J_{9,7}=1.4 \mathrm{~Hz}, J_{6,7}=\right.$ $3.0 \mathrm{~Hz} ; 7-\mathrm{H}), 3.1-3.2(\mathrm{~m} ; 10-\mathrm{H}, 12-\mathrm{H}), 3.18(\mathrm{~m} ; 9-\mathrm{H}), 3.26(\mathrm{~m} ; 1-\mathrm{H}), 4.41$ (dd; 3-H), 5.07 (t, $\left.J_{5,6}=3.0 \mathrm{~Hz} ; 6-\mathrm{H}\right), 6.0-6.1(\mathrm{~m} ; 13-\mathrm{H}, 14-\mathrm{H}), 6.44(\mathrm{dd} ; 5-\mathrm{H}) .-{ }^{13} \mathrm{C}-\mathrm{NMR}\left(\mathrm{CDCl}_{3}\right): \delta=35.7$, 36.6 (jeweils d, 141.0 bzw. $139.5 \mathrm{~Hz}$; C-1, C-9); 45.0, 45.1, 45.6 (jeweils d, 144, 144 bzw. $139.5 \mathrm{~Hz}$; C-2, C-7, C-8); $46.6,47.6$ (jeweils d, 186.0 bzw. $186.5 \mathrm{~Hz} ; \mathrm{C}-10, \mathrm{C}-12$ ); 82.0 (d, $159.9 \mathrm{~Hz}$; C-3); 103.7 (d, 175.8 Hz; C-6); 127.9, 128.1 (jeweils d, $164.2 \mathrm{~Hz}$; C-13, C-14); 147.4 (d, $194.7 \mathrm{~Hz} ; \mathrm{C}-5)$.

$$
\mathrm{C}_{12} \mathrm{H}_{12} \mathrm{O}_{2} \text { (188.2) Ber. C } 76.58 \text { H } 6.43 \text { Gef. C } 75.93 \text { H } 6.83
$$

c) In der Gasphase: Ca. $160 \mathrm{mg} 18$ in $0.5 \mathrm{ml} \mathrm{C}_{6} \mathrm{D}_{6}$ wurden unter Ruhren und schwachem Erwärmen bei 14 Torr durch ein $20 \mathrm{~cm}$ langes, mit Quarzscherben gefulltes und $250^{\circ} \mathrm{C}$ heißes Quarzrohr in eine auf $-78^{\circ} \mathrm{C}$ gekülte Vorlage destilliert. Die quantitative NMR-Analyse zeigte, daß 53\% 18 unverändert geblieben und 7\% in 20/21 ubbergegangen waren. Bezogen auf eingesetztes 18 betrug die Ausbeute an 19 ca. $0.2 \%$ (d.h. 20/21: 19 35:1).

Thermolyse von 2-Oxabicyclo/3.2.0/hepta-3,6-dien (19): Ca. $50 \mathrm{mg} 19$ in $0.5 \mathrm{ml} \mathrm{C}_{6} \mathrm{D}_{6}$, verunreinigt mit $\mathrm{CHCl}_{3}$-Spuren, wurden in einem NMR-Rohr durch mehrere Einfrieren (fl. $\mathrm{N}_{2}$ )Abpumpen $\left(10^{-3}\right.$ Torr)-Auftauen-Zyklen entgast und unter Vak. eingeschmolzen. Bei $150^{\circ} \mathrm{C}$ dauerte es $9.5 \mathrm{~h}$ bis 19 vollständig abgebaut war. Die neuen Banden im NMR-Spektrum stammten eindeutig von 20/21. Signale von Phenol traten nicht auf.

Thermolyse von 18a: Die dideuterierte Verbindung $18 \mathrm{a}$ wurde in ähnlicher Verdünnung in $\mathrm{C}_{6} \mathrm{D}_{6}$ wie 18, Versuch a), thermolysiert; $\mathrm{CHCl}_{3}$ und Cyclohexan waren hier nicht anwesend. Im NMR-Spektrum des umgelagerten Produkts sind die Intensităten der Banden bei $\delta=3.44$ und 6.34 stark reduziert und die Multiplizităten der anderen Absorptionen dementsprechend verăndert, d.h. die Deuteriumatome sind hauptsăchlich in den Positionen 5 und 6 gelandet, wie in 19a dargestellt. Allerdings sind die Restintensitäten stărker als aufgrund des Deuterierungsgrads von 18 a erwartet, so daß möglicherweise neben 19 a noch ein anderes isotopomeres aus 18 a gebildet wird.

endo, endo-5.(Phenylthio)tricyclo/2.2.0.0 2.6 Jhexan-3-ol (28): Nachdem zwischen $610 \mathrm{mg}$ (6.50 mmol) 18, verunreinigt mit Resten von $\mathrm{CHCl}_{3}$ und THF, und $720 \mathrm{mg}(6.50 \mathrm{mmol})$ Thiophenol in $5 \mathrm{ml}$ absol. Benzol bei $20^{\circ} \mathrm{C}$ innerhalb von $2 \mathrm{~d}$ keine Reaktion eingetreten war, bestrahlte man das Gemisch bei Raumtemp. $42 \mathrm{~h}$ mit 350-nm-Licht (Gräntzel-Reaktor 400). Nach dem Einengen, zuletzt im 0.01-Torr-Vak., hinterblieben $1.02 \mathrm{~g}(76 \%)$ einer braunen, viskosen Flüssigkeit, die aufgrund des NMR-Spektrums hauptsächlich aus 28 bestand und wahrscheinlich auch trans-4-(Phenylthio)tricyclo/3.1.0.0.6.6 Jhexan-3-ol (27) enthielt (Verhältnis $27: 28=5: 95)$. Daneben vorhandene Verunreinigungen konnten auch durch Destillation bei $55-65^{\circ} \mathrm{C}(\mathrm{Bad}) / 10^{-3}$ Torr nicht abgetrennt werden, obwohl sich dabei die Farbe nach Gelb aufhellte. - ${ }^{1} \mathrm{H}-\mathrm{NMR}\left(\mathrm{CDCl}_{3}\right): 28: \delta=$ $2.1-2.4\left(\mathrm{~m} ; 2-\mathrm{H}, 6-\mathrm{H}\right.$ ), 2.50 (td, $J_{1,2}=J_{1,6}=3.9 \mathrm{~Hz}, J_{1,4}=1.2 \mathrm{~Hz} ; 1-\mathrm{H}$ ), 2.70 (quint d, $J_{2.4}=$ $\left.J_{3,4}=J_{4,5}=J_{4,6}=4.0 \mathrm{~Hz} ; 4-\mathrm{H}\right), 4.38\left(\mathrm{dd}, J_{5,6}=2.2 \mathrm{~Hz} ; 5-\mathrm{H}\right) ; \approx 4.3(\mathrm{~m} ; 3-\mathrm{H}), 4.60\left(\mathrm{~d}, J_{3, \mathrm{OH}}=\right.$ $10.8 \mathrm{~Hz} ; \mathrm{OH}$, dieses Signal verschwindet beim Schütteln mit $\left.\mathrm{D}_{2} \mathrm{O}\right), 7.0-7.6\left(\mathrm{~m} ; \mathrm{C}_{6} \mathrm{H}_{5}\right)$. -27 : $\delta=3.32$ und 3.78 (jeweils br.s; $3-\mathrm{H}, 4-\mathrm{H}$ ).

Chem. Ber. $117(1984)$ 
endo,endo-5-Acetoxytricyclo[2.2.0.0.6.6 /hexan-3-ol (29) und anti-6-Acetoxy-endo-bicyclo[2.1.1]hex-2-en-5-ol (30): $\mathrm{Zu} 540 \mathrm{mg}$ (5.75 mmol) 18 in $2 \mathrm{ml}$ absol. Benzol gab man bei Raumtemp. tropfenweise $276 \mathrm{mg}$ Essigsäure $(4.60 \mathrm{mmol})$ in $3 \mathrm{ml}$ absol. Benzol. $10 \mathrm{~min}$ nach vollendeter Zugabe zeigte ein ${ }^{1} \mathrm{H}$-NMR-Spektrum neben wenig 18 nur das Vorliegen von 29 an. Das Abdampfen des Benzols bei $0^{\circ} \mathrm{C} / 14$ Torr hinterließ $517 \mathrm{mg}(73 \%)$ einer braunen, viskosen Flüssigkeit, die aufgrund der NMR-Analyse aus 29 und 30 im Verhalltnis $92: 8$ bestand. Die Destillation eines kleinen Anteils bei $30-40^{\circ} \mathrm{C}(\mathrm{Bad}) / 10^{-3}$ Torr lieferte ein farbloses $1: 1$-Gemisch aus 29 und 30 . ${ }^{1} \mathrm{H}-\mathrm{NMR}\left(\mathrm{CDCl}_{3}\right): 29: \delta=2.01\left(\mathrm{~s} ; \mathrm{CH}_{3}\right), 2.27-2.55(\mathrm{~m} ; 1-\mathrm{H}, 2-\mathrm{H}, 6-\mathrm{H}), 3.01$ (quint d, $J_{2.4}=$ $J_{3,4}=J_{4,5}=J_{4,6}=4.3 \mathrm{~Hz}, J_{1,4}=1.3 \mathrm{~Hz} ; 4-\mathrm{H}$ ), 3.83 (breit; OH), $4.32(4.3-\mathrm{Hz}-\mathrm{d}$ von $1.5-\mathrm{Hz}$ pseudo t; 3-H), $5.26\left(4.3-\mathrm{Hz}\right.$-d von 1.5-Hz-pseudo t; 5-H). 30: $\delta=2.06$ (s; $\mathrm{CH}_{3}$ ), 3.00 (pseudo q, Linienabstand $2.3 \mathrm{~Hz} ; 1,4-\mathrm{H}), 4.61(\mathrm{~s} ; 6-\mathrm{H}), 5.28\left(\mathrm{t}, J_{1.5}=2.4 \mathrm{~Hz} ; 5-\mathrm{H}\right), 6.58$ (pseudo t, Linienabstand $2.1 \mathrm{~Hz} ; 2,3-\mathrm{H})$.

Bei verschiedenen Ansätzen beobachtėten wir neben 30 ein weiteres Umlagerungsprodukt in wechselnden, zum Teil nicht unerheblichen Mengen. Seine ${ }^{1} \mathrm{H}-\mathrm{NMR}-$ Banden, u. a. je ein 2.3-Hz-1 bei $\delta=5.08$ und 6.53 sprechen fur die Bicyclo[2.1.1]hex-2-en-Konstitution. Moglicherweise konkurrieren Alkoholfunktionen mit dem Acetation um 12, so daß Ether mit zwei $\mathrm{C}_{6}$-Einheiten entstehen.

exo-6-Acetoxy-exo-bicyclo/3.1.0/hex-2-en-4-ol (31): $\mathrm{Zu} 300 \mathrm{mg}$ (3.19 mmol) 18 in $0.5 \mathrm{ml} \mathrm{THF}$ tropfte man bei $0^{\circ} \mathrm{C} 191 \mathrm{mg}$ ( $3.19 \mathrm{mmol}$ ) Essigsäure, verdampfte das THF bei 15 Torr, löste den Rutckstand in $5 \mathrm{ml}$ Benzol und schmolz die farblose Losung unter Vakuum in eine Ampulle und einen kleinen Anteil zur NMR-Kontrolle in ein NMR-Rohr ein. Nach $1 \mathrm{~h}$ bei $130^{\circ} \mathrm{C}$ waren die Banden von 30 vollständig verschwunden. Die vereinigten Lösungen wurden im Vak. eingeengt, und aus dem Rückstand destillierten bei $40-80^{\circ} \mathrm{C}$ (Bad) $/ 10^{-3}$ Torr $300 \mathrm{mg}(61 \%) 31$ als farblose Flüssigkeit. Für die Elementaranalyse wurde erneut destilliert und die Fraktion mit Sdp. $50-55^{\circ} \mathrm{C}(\mathrm{Bad}) / 10^{-3}$ Torr untersucht. - IR (Film): 3390 (breit, OH), $1752 \mathrm{~cm}^{-1}(\mathrm{C}=\mathrm{O})$. MS (70 eV): $m / e=112(18 \%), 94(7), 83(9), 66(56), 55(7), 43$ (100), 39 (10). - 'H-NMR ( $\left[D_{6}\right.$ IDMSO): $\delta=1.83$ und 2.10 (jeweils dm, $\left.J_{1,5}=6.5 \mathrm{~Hz} ; 1-\mathrm{H}, 5-\mathrm{H}\right), 1.97\left(\mathrm{~s} ; \mathrm{CH}_{3}\right), 3.00(\mathrm{~m}$; 6-H), $4.34(\mathrm{~m} ; 4-\mathrm{H}), 4.95\left(\mathrm{~d}, J_{4 . \mathrm{OH}}=6.4 \mathrm{~Hz} ; \mathrm{OH}\right), 5.58$ und 5.94 (jeweils $\mathrm{dm}, J_{2,3}=5.4 \mathrm{~Hz}$; 2-H, 3-H). $-{ }^{13} \mathrm{C}-\mathrm{NMR}\left(\mathrm{CDCl}_{3}\right): \delta=20.7\left(\mathrm{CH}_{3}\right), 29.4$ und 30.5 (C-1 und C-5), $60.4(\mathrm{C}-6), 75.6$ $(C-4), 133.0$ und $134.1(C-2$ und $C-3), 170.9(C=0)$.

$$
\mathrm{C}_{8} \mathrm{H}_{10} \mathrm{O}_{3} \text { (154.2) Ber. C } 62.30 \text { H } 6.54 \text { Gef. C } 61.41 \text { H } 6.85
$$

Tricyclo/3.1.0.0.6 Jhexan-3-ol (32) und endo-Tricyclo/2.2.0.0.0.6 Jhexan-3-ol (33): Die Losung von $1.72 \mathrm{~g}(18.3 \mathrm{mmol}) 18$ und $1 \mathrm{ml}$ THF in $40 \mathrm{ml}$ absol. Ether wurde unter Stickstoff innerhalb von $20 \mathrm{~min}$ zur siedenden Suspension von $1.2 \mathrm{~g}(31.8 \mathrm{mmol}) \mathrm{LiAlH}_{4}$ in $30 \mathrm{ml}$ Ether getropft. Man kochte $3 \mathrm{~h}$ unter Rückfluß, kuhlte dann auf $0^{\circ} \mathrm{C}$, hydrolysierte vorsichtig, filterierte den Niederschlag der Hydroxide ab, trocknete das Filtrat mit $\mathrm{K}_{2} \mathrm{CO}_{3} / \mathrm{Na}_{2} \mathrm{SO}_{4}$ und engte i. Vak. ein. Aus dem Rückstand destillierten bei $60-80^{\circ} \mathrm{C}(\mathrm{Bad}) / 14$ Torr $1.23 \mathrm{~g}(70 \%)$ einer farblosen oligen Flüssigkeit, deren 'H-NMR-Spektrum sie als $1: 1$-Gemisch aus 32 und 33 charakterisierte. Korrekte Elementaranalysenwerte wurden nicht erhalten, obwohl die NMR-Spektren kaum Verunreinigungen anzeigten.

Bei ahnlichen Ausbeuten änderte sich das Isomerenverhaltınis, als die Reduktion bei tieferer Temperatur oder in THF durchgeführt wurde:

\begin{tabular}{|c|c|c|}
\hline Loosungsmittel & Temperatur & $32: 33$ \\
\hline Ether & $\begin{array}{l}\text { Zutropfen bei }-50^{\circ} \mathrm{C} \text {, dann } 3 \mathrm{~h}-50 \text { bis } 0^{\circ} \mathrm{C} \text {, } \\
5 \mathrm{~h} 0^{\circ} \mathrm{C}\end{array}$ & $3: 1$ \\
\hline THF & $\begin{array}{l}\text { Zutropfen bei }-50^{\circ} \mathrm{C} \text {, dann } 3 \mathrm{~h}-50 \text { bis } 0^{\circ} \mathrm{C} \text {, } \\
5 \mathrm{~h} 0^{\circ} \mathrm{C}\end{array}$ & $>10: 1$ \\
\hline THF & $66^{\circ} \mathrm{C}$ & $4: 1$ \\
\hline
\end{tabular}


Bei der Reduktion mit Lithiumtriethylborhydrid in THF entsprechend der Vorschrift in Lit. ${ }^{43)}$ wurde das Reagens bei $-20^{\circ} \mathrm{C}$ innerhalb von 30 min zu 18 getropft. Nach 1 h Rückflußkochen erhielt man mit $41 \%$ Ausbeute 32 und 33 im Verhăltnis $1: 1$.

$\mathrm{Zu}$ Natriumdiethylaluminiumhydrid (28proz. Losung in Toluol) wurde 18 (10proz. Lossung in Ether) bei $0^{\circ} \mathrm{C}$ getropft und dann $5 \mathrm{~h}$ bei $0^{\circ} \mathrm{C}$ geruhrt. Aufarbeitung wie bei den $\mathrm{LiAlH}_{4}$ Versuchen: $42 \% 32$ und 33 im Verhaltnis 1.5: 1.

32: ${ }^{1} \mathrm{H}-\mathrm{NMR}\left(\mathrm{CDCl}_{3}\right): \delta=1.04$ (br.d, $\left.J_{4 \text {-is, } 4 \text { trans }}=12.0 \mathrm{~Hz} ; 4 \mathrm{H}_{\text {cis }}\right), 1.24$ (breit; $\left.\mathrm{OH}\right), 1.77$ (dddt, $J_{3,4 \text { trans }}=7.1 \mathrm{~Hz}, J_{4 \text { trans }, 6}=1.5 \mathrm{~Hz}, J_{1,4 \text { trans }}=J_{4 \text { trans, } 5}=0.8 \mathrm{~Hz} ; 4-\mathrm{H}_{\text {trans }}$ ), 1.92 (dqd, $J_{1,6}=9.3 \mathrm{~Hz}_{1} J_{1,2}=J_{1,4 \text { cis }}=J_{1,5}=1.8 \mathrm{~Hz} ; 1-\mathrm{H}$ ), $2.03(\mathrm{~m} ; 5-\mathrm{H}$ ); 2.05 (br.d; 6-H); 2.25 (dq, $\left.J_{2,5}=4.8 \mathrm{~Hz} ; J_{2,3}=J_{2,6}=1.7 \mathrm{~Hz} ; 2-\mathrm{H}\right), 4.15$ (br.d; 3-H). $-{ }^{13} \mathrm{C}-\mathrm{NMR}:$ Tab. 1 .

33: ' $\mathrm{H}-\mathrm{NMR}\left(\mathrm{CDCl}_{3}\right): \delta=1.80(\mathrm{~m} ; 6-\mathrm{H}), 1.86\left(\mathrm{~m} ; 5-\mathrm{H}_{\text {endo }}\right), 1.97\left(\mathrm{br} . \mathrm{d}, J_{3, \mathrm{OH}} \approx 6.5 \mathrm{~Hz} ; \mathrm{OH}\right)$, $2.17(\mathrm{~m} ; 2-\mathrm{H}), 2.5 t-2.58\left(\mathrm{~m} ; 1-\mathrm{H}, 4-\mathrm{H}, 5-\mathrm{H}_{\mathrm{c}, \mathrm{ro}}\right), 4.37$ (m; 3-H). - ${ }^{13} \mathrm{C}-\mathrm{NMR}:$ Lit. ${ }^{53)}$.

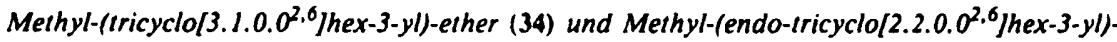
ether (35): Zur geruhrten Losung von $1.43 \mathrm{~g}$ (14.9 mmol) $1: 1$-Gemisch aus 32 und 33 sowie 3.79 ( $26.7 \mathrm{mmol}$ ) Methyliodid in $19 \mathrm{ml}$ absol. THF fügte man bei Raumtemp. innerhalb von $30 \mathrm{~min}$ portionsweise $640 \mathrm{mg}$ ( $26.7 \mathrm{mmol})$ Natriumhydrid. Unter leichtem Erwärmen färbte sich das Gemisch bräunlich. Nach dem $\mathrm{NaH}$ gab man noch einmal $3.79 \mathrm{~g}$ Methyliodid $2 \mathrm{u}$, rührte $2 \mathrm{~h}$ bei $20^{\circ} \mathrm{C}$ und kondensierte dann Losungsmittel und Produkte i. Vak., zuletzt bei $0.01 \mathrm{Torr}$, in eine auf $-78^{\circ} \mathrm{C}$ gekuhlte Vorlage um. Das THF des Kondensats wurde weitgehend über eine Vigreuxkolonne bei Normaldruck und dann bei $20^{\circ} \mathrm{C} / 14$ Torr abdestilliert. Aus dem Ruckstand kondensierten bei $20^{\circ} \mathrm{C} / 0.001$ Torr $1.35 \mathrm{~g}$ einer farblosen Flussigkeit, die aufgrund der NMR-Analyse $575 \mathrm{mg}(69 \%) 34,430 \mathrm{mg}(52 \%) 35$ und daneben THF enthielt. Dieses Gemisch wurde durch präparative Gaschromatographie getrennt. Säule: $6 \mathrm{~m}, 5 \% \mathrm{NaOH}$ und $20 \%$ Carbowax $20 \mathrm{M}$ auf Chromosorb $\mathrm{P}, 80^{\circ} \mathrm{C} ; 35$ hat die kürzere und 34 die längere Retentionszeit.

34: IR (Film): 3115 (w), 3050 (m), 2978 (m), 2938 (s), 2877 (s), 2820 (m), $1460(\mathrm{w}), 1403$ (m), $1351(\mathrm{~m}), 1346(\mathrm{~m}), 1282(\mathrm{w}), 1248(\mathrm{w}), 1224(\mathrm{w}), 1208(\mathrm{~m}), 1148(\mathrm{~m}), 1127(\mathrm{~m}), 1100(\mathrm{~s}), 1090(\mathrm{~s})$, $1051(\mathrm{~m}), 1029(\mathrm{~m}), 959(\mathrm{~m}), 880(\mathrm{w}), 845(\mathrm{w}), 753(\mathrm{~s}) \mathrm{cm}^{-1}$. MS $(70 \mathrm{eV}): \mathrm{m} / \mathrm{e}=110(44 \%$, $\mathrm{M}^{+}$), $109(68), 95(91), 94(15), 80(42), 79\left(100, \mathrm{M}-\mathrm{OCH}_{3}\right), 78(91), 77(91), 67(88), 66(16), 65$ (28), $53(16), 52(59), 51$ (46), $50(27), 45$ (47), 41 (87), 39 (81). - ' $\mathrm{H}$-NMR: Tab. 3. - ${ }^{13} \mathrm{C}-\mathrm{NMR}$ : Tab. 2.

35: IR (Film): 3095 (w), 3062 (s), 3000 (s), 2980 (s), 2930 (s), 2880 (s), 2820 (s), 1460 (m), 1362 $(\mathrm{s}), 1327(\mathrm{~m}), 1305(\mathrm{~m}), 1250(\mathrm{~m}), 1230(\mathrm{~m}), 1210(\mathrm{~m}), 1202(\mathrm{~s}), 1192(\mathrm{~s}), 1144(\mathrm{~m}), 1130(\mathrm{~s}), 1105$ (s), $1052(\mathrm{~s}), 1035(\mathrm{~m}), 1020(\mathrm{~m}), 1000(\mathrm{~m}), 978(\mathrm{~m}), 940(\mathrm{~m}), 890(\mathrm{w}), 867(\mathrm{w}), 826(\mathrm{w}), 810(\mathrm{~m})$, $784(\mathrm{~m}), 770(\mathrm{~m}), 724(\mathrm{w}), 716(\mathrm{w}) \mathrm{cm}^{-1}$. $-\mathrm{MS}(70 \mathrm{eV}): \mathrm{m} / \mathrm{e}=110\left(50 \%, \mathrm{M}^{+}\right), 109(57), 95(41)$, $94(12), 80(22), 79\left(93, \mathrm{M}-\mathrm{OCH}_{3}\right), 78(41), 67(82), 66(19), 65(31), 53(14), 52(17), 51(30), 50$ (16), 45 (65), 41 (100), 40 (14), 39 (85). - 'H-NMR: Tab. 4. - ${ }^{13} \mathrm{C}-N M R:$ Lit. ${ }^{55)}$.

$$
\mathrm{C}_{7} \mathrm{H}_{10} \mathrm{O} \text { (110.2) Ber. C } 76.33 \mathrm{H} 9.15 \text { 34: Gef. C } 76.11 \text { H } 9.25
$$

35: Gef. C 76.05 H 9.20

1) Aus der Dissertation $H$. Leininger, Univ. Würzburg 1981.

2) R. Gleiler, Top. Curr. Chem. 86, 197 (1979).

3) M. Christl, Angew. Chem. 93, 515 (1981); Angew. Chem., Int. Ed. EngI. 20, 529 (1981).

4) $H$. Leininger, F. Lanzendorfer und M. Christl, Chem. Ber. 116, 669 (1983).

5) H. Leininger, M. Christl und D. Wendisch, Chem. Ber. 116, 681 (1983).

6) Ein Teil dieser Ergebnisse war Gegenstand einer Kurzmitteilung: $H$. Leininger und $M$. Christl, Angew. Chem. 92, 466 (1980); Angew. Chem., Int. Ed. Engl. 19, 458 (1980), und wurde auch im Rahmen einer Ubersicht ${ }^{3)}$ zitiert.

7) K. B. Sharpless und K. Akashi, J. Am. Chem. Soc. 98, 1986 (1976); K. Akashi, R. E. Palermo und $K$. B. Sharpless, J. Org. Chem. 43, 2063 (1978).

8) P. Bischof, R. Gleiter und E. Müller, Tetrahedron 32, 2769 (1976).

Chem. Ber. $117(1984)$ 
9) A. Schloßer, F. Philipp, W. Mickler, G. Szeimies und H.-D. Martin, Chem. Ber. 113, 1053 (1980).

10) G. Szeimies, A. Schloßer, F. Philipp, P. Dietz und W. Mickler, Chem. Ber. 111, 1922 (1978).

11) R. Herbert und M. Christl, Chem. Ber. 112, 2012 (1979).

12) K. B. Wiberg, B. R. Lowry und B. J. Nist, J. Am. Chem. Soc. 84, 1594 (1962).

13) G. Szeimies und P. Dietz, Chem. Ber. 113, 398 (1980).

14) $H$. Günther, NMR-Spektroskopie, 2. Aufl., Thieme, Stuttgart 1983. - 14a) S. $112 \mathrm{ff}$. 14b) S. $105 \mathrm{ff}$.

15) In supersaurem Medium sind derartige Kationen stabil und haben die nicht klassische Struktur: 15a) S. Masamune, E. N. Cain, R. Vukov, S. Takada und Nakatsuka, Chem. Commun. 1969, 243. - 15b) S. Masamune, S. Takada, N. Nakatsuka, R. Vukov und E. N. Cain, J. Am. Chem. Soc. 91, 4322 (1969). - isc) $H$. Hogeveen und $P$. W. Kwant, J. Am. Chem. Soc. 95, 7315 (1973).

16) 16a) $R$. J. Roth und T. J. Katz, J. Am. Chem. Soc. 94, $4770(1972) .-{ }^{16 b)}$ R. J. Roth und T. J. Katz, J. Org. Chem. 45, 961 (1980).

17) $H$. Leininger, P. Kemmer, K. Beck und $M$. Chrisll, Chem. Ber. 115, 3213 (1982).

18) M. S. Newman und C. H. Chen, J. Am. Chem. Soc. 94, 2149 (1972); J. Org. Chem. 38, 1173 (1973).

19) 19a) H. Yagi und D. M. Jerina, J. Am. Chem. Soc. 95, 243 (1973). - 196) P. Dansette und D. M. Jerina, J. Am. Chem. Soc. 96, 1224 (1974).

${ }^{20)} M$. J. Robins, R. Mengel und R. A. Jones, J. Am. Chem. Soc. 95, 4074 (1973).

21) $M$. S. Newman und D. R. Olson, J. Org. Chem. 38, 4203 (1973).

22) M. S. Newman und C. H. Chen, J. Am. Chem. Soc. 95, 278 (1973).

23) E. Hoft und S. Ganschow, J. Prakt. Chem. 314, 145 (1972).

24) J. Rebek jr., S. F. Wolf und A. B. Mossman, J. Chem. Soc., Chem. Commun. 1974, 711; J. Rebek, R. McCready, S. Wolf und A. Mossmann, J. Org. Chem. 44, 1485 (1979).

25) E. P. Kyba und D. C. Alexander, Tetrahedron Lett. 1976, 4563.

26) $R$. J. Rolh, Synt. Commun. 9, 751 (1979).

27) E. Pretsch, T. Clerc, J. Seibl und W. Simon, Tabellen zur Strukturaufklärung organischer Verbindungen mit spektroskopischen Methoden, Springer Verlag, Berlin, Heidelberg, New York 1976. - 27a) S. HS. - 270) S. C 70.

28) E. Vogel, W. A. Böll und $H$. Günther, Tetrahedron Lett. 1965, 609.

29) $E$. Vogel und $H$. Günther, Angew. Chem. 79, 429 (1967); Angew. Chem., Int. Ed. Engl. 6, 385 (1967).

30) J. M. Holouka und P. D. Gardner, J. Am. Chem. Soc. 89, 6390 (1967).

31) A. G. Anastassiou, E. Reichmanis, S. J. Girgenti und M. Schaefer-Ridder, J. Org. Chem. 43, 315 (1978).

32) M. J. Goldstein, M. W. Johnson und R. T. Taylor, Tetrahedron Lett. 23, 3331 (1982).

33) W. H. Rastetter, J. Am. Chem. Soc. 97, 210 (1975).

34) $R$. B. Woodward und $R$. Hoffmann, Angew. Chem. 81,797 (1969); Angew. Chem., Int. Ed. Engl. 8, 781 (1969).

35) L. A. Paquette und J. H. Barrett, J. Am. Chem. Soc. 88, 1718 (1966).

36) M. Christl, U. Heinemann und W. Kristof, J. Am. Chem. Soc. 97, 2299 (1975).

37) $M$. Christl und $H$. Leininger, Tetrahedron Lett. 1979, 1553.

38) J. F. Ireland und P. A. H. Wyatt, Adv. Phys. Org. Chem. 12, 131 (1976).

39) T. J. Katz und N. Acton, J. Am. Chem. Soc. 95, 2738 (1973).

40) T. J. Katz und K. C. Nicolaou, J. Am. Chem. Soc. 96, 1948 (1974).

41) $M$. Christl, E. Brunn und $F$. Lanzendorfer, J. Am. Chem. Soc. 106, 373 (1984).

42) 42a) $F . T$. Bond und $L$. Scerbo, Tetrahedron Lett. 1968, 2789. - 42b) $H$. M. Frey und $R . G$. Hopkins, J. Chem. Soc. B 1970, 1410. - 42c) W. R. Roih und $A$. Friedrich, Tetrahedron Lell. 1969, 2607. - 42d' F. Scheidt und W. Kirmse, J. Chem. Soc., Chem. Commun. 1972, 716.

43) E. Müller, Chem. Ber. 108, 1394 (1975).

44) $H$. Leininger, unveröffentlichte Versuche.

45) S. Krishnamurthy, R. M. Schubert und H. C. Brown, J. Am. Chem. Soc. 95, 8486 (1973), und dort zitierte Literatur.

46) R. D. Suenram, J. Am. Chem. Soc. 97, 4869 (1975).

47) $W$. Kirmse und $F$. Scheidt, Angew. Chem. 83, 251 (1971); Angew. Chem., Int. Ed. Engl. 10, 263 (1971).

48) $H$. Jendralla, Chem. Ber. 113, 3585 (1980).

49) D. M. Lemal und K. S. Shim, J. Am. Chem. Soc. 86, 1550 (1964).

so) W. R. Moore, S. S. Hall und C. Largman, Tetrahedron Lett. 1969, 4353.

Chem. Ber. $117(1984)$ 
51) M. Christl und G. Brüntrup, Chem. Ber. 107, 3908 (1974).

52) M. Christl, H.-J. Lüddeke, A. Nagyrevi-Neppel und G. Freitag, Chem. Ber. 110, 3745 (1977). Die Triplettaufspaltung von ca. $8 \mathrm{~Hz}$, die bei Diels-Alder-Addukten von $1 \mathrm{im}$ Signal des Bicyclo[1.1.0]butan-Brückenkopf-C-Atoms auftritt, der cis zum anellierten Ring steht, ist nicht zwei geminalen, sondern zwei vicinalen $\mathrm{CH}$-Kopplungen zuzuordnen (vgl. Diskussion im Abschnitt F.1.).

53) J. L. Marshall, Carbon-Carbon and Carbon-Proton NMR Couplings, Verlag Chemie International, Deerfied Beach 1983.

54) M. Christl, H. Leininger und E. Brunn, J. Org. Chem. 47, 661 (1982).

55) $M$. Christl, H. Leininger und B. Mattauch, Spectrosc. Int. J. 2, $18+(1983)$.

56) T. J. Katz, E. J. Wang und N. Acton, J. Am. Chem. Soc. 93. $3782(1971) ; T . J . K a t z, R . J$. Roth, N. Acton und E. J. Carnahan, Org. Synth. 53, 157 (1973).

57) Zur Darstellung von Benzvalen verwendeten wir bromidfreies Methyllithium von CHEMETALL, Frankfurt am Main.

$[335 / 83]$ 